\title{
THE AIR TRAFFIC FLOW MANAGEMENT PROBLEM WITH ENROUTE CAPACITIES
}

\author{
DIMITRIS BERTSIMAS and SARAH STOCK PATTERSON
}

\author{
Massachusetts Institute of Technology, Cambridge, Massachusetts \\ (Received August 1994; revision received October 1995; accepted May 1996)
}

\begin{abstract}
Throughout the United States and Europe, demand for airport use has been increasing rapidly, while airport capacity has been stagnating. Over the last ten years the number of passengers has increased by more than 50 percent and is expected to continue increasing at this rate. Acute congestion in many major airports has been the unfortunate result. For U.S. airlines, the expected yearly cost of the resulting delays is currently estimated at $\$ 3$ billion. In order to put this number in perspective, the total reported losses of all U.S. airlines amounted to approximately $\$ 2$ billion in 1991 and $\$ 2.5$ billion in 1990 . Furthermore, every day 700 to 1100 flights are delayed by 15 minutes or more. European airlines are in a similar plight. Optimally controlling the flow of aircraft either by adjusting their release times into the network (ground-holding) or their speed once they are airborne is a cost effective method to reduce the impact of congestion on the air traffic system. This paper makes the following contributions: (a) we build a model that takes into account the capacities of the National Airspace System (NAS) as well as the capacities at the airports, and we show that the resulting formulation is rather strong as some of the proposed inequalities are facet defining for the convex hull of solutions; (b) we address the complexity of the problem; (c) we extend that model to account for several variations of the basic problem, most notably, how to reroute flights and how to handle banks in the hub and spoke system; (d) we show that by relaxing some of our constraints we obtain a previously addressed problem and that the LP relaxation bound of our formulation is at least as strong when compared to all others proposed in the literature for this problem; and (e) we solve large scale, realistic size problems with several thousand flights.
\end{abstract}

$\mathrm{T}$ hroughout the United States and Europe, demand for airport use has been increasing rapidly during recent years, while airport capacity has been stagnating. Over the last ten years the number of passengers has increased by more than 50 percent and is expected to continue increasing at this rate, while no appreciable increase in capacity is expected. Acute congestion in many major airports has been the unfortunate result. For U.S. airlines, the expected yearly cost of the resulting delays is currently estimated at $\$ 3$ billion. In order to put this number in perspective, the total reported losses of all U.S. airlines amounted to approximately $\$ 2$ billion in 1991 and $\$ 2.5$ billion in 1990 . Furthermore, every day 700 to 1100 flights are delayed by 15 minutes or more. European airlines are in a similar plight. Thus, congestion is a problem of undeniable practical significance.

Faced with the realities of congestion, the FAA has been using ground-holding policies to reduce delay costs. These short-term policies consider airport capacities and flight schedules as fixed for a given time period, and adjust the flow of aircraft on a real time basis by imposing "ground holds" on certain flights. Such a flight is then held on the ground at its departure airport even if it is otherwise ready for takeoff. Ground-holding makes sense in the following situation. Suppose it has been determined that if an aircraft departs on time, then it will encounter congestion, incurring an airborne delay as it awaits landing clearance at its destination airport. However, by delaying its departure, the aircraft will arrive at its destination at a later time when minimal congestion is expected, thus, in- curring no airborne delay. Therefore, the objective of ground-holding policies is to "translate" anticipated airborne delays to the ground.

The effectiveness of ground-holding policies lies in the following two fundamental facts. First, while a flight is airborne it incurs costs such as fuel and safety costs that are not applicable before the flight takes off. These costs make airborne delays much costlier than ground delays. Second, airport capacity is highly variable due to its heavy dependence on the weather (visibility, wind, precipitation, cloud ceiling). It is not unusual for the capacity of an airport to be reduced by 50 percent in inclement weather. Given these two facts, there is significant potential to reduce costs when adjusting aircraft flow as weather (hence airport capacity) forecasts change in such a way that ground delays are substituted for the much costlier airborne delays.

Currently, the FAA implements a national groundholding policy. This policy uses a computerized procedure based on a first-come, first-served rule, in order to select appropriate ground-holds. These selections are further enhanced through the experience of its air traffic controllers. In the last decade, several models have been developed that use optimization techniques to improve upon current practices. We will briefly review these developments.

A Taxonomy of Models. In Odoni (1987), the problem of scheduling flights in real time in order to minimize congestion costs was first conceptualized and introduced. Since 
then several models have been proposed for solving different versions of this problem. The first and simplest version considers a single airport and makes decisions about the ground-holds for this Single-Airport Problem (SAGHP). The Multi-Airport Ground-Holding Problem (MAGHP) was the next problem to be introduced. It makes groundholding decisions for an entire network of airports. Thus, the SAGHP and the MAGHP are distinguished by whether delays are assumed to propagate in the network of airports as aircraft perform consecutive flights. Besides determining release times for aircraft (ground-holding), the Air Traffic Flow Management Problem (TFMP) also determines the optimal speed adjustment of aircraft while airborne for a network of airports taking into account the capacitated airspace. Thus, the TFMP determines how to control a flight throughout its duration, not simply before its departure. If we add the final complication, rerouting of flights due to drastic fluctuations in the available capacity of airspace regions, we obtain the Air Traffic Flow Management Rerouting Problem (TFMRP). In this problem, a flight may be rerouted through a different flight path in order to reach its destination if the current route passes through a region that is unusable for reasons usually related to poor weather conditions. In order to describe the work on these problems we consider the following modeling variations:

1. Deterministic vs. stochastic models, which are distinguished by whether the capacities of the system (airports and sectors in the airspace) are assumed deterministic or probabilistic.

2. Static vs. dynamic models, which are distinguished by whether or not the solutions are updated dynamically during the day.

The deterministic SAGHP (both static and dynamic) was first formulated as a network flow problem in Terrab and Odoni (1991). The stochastic SAGHP was formulated and solved as a stochastic programming problem in Richetta and Odoni (1993) (the static case) and Richetta and Odoni (1994) (the dynamic case). A review of optimization models for the SAGHP is given in Andreatta et al. (1993). The deterministic MAGHP was formulated as a 0-1 integer programming problem in Vranas et al. (1994a) (the static case) and in Vranas et al. (1994b) (the dynamic case). Terrab and Paulose (1993) address the stochastic MAGHP as a stochastic programming problem.

In this paper we present a $0-1$ integer programming model for the deterministic, multiairport TFMP that addresses capacity restrictions on the en route airspace. Simultaneously with our work, models addressing enroute capacities were also introduced by Lindsay et al. (1993). They propose integer programming formulations for a version of TFMP that tracks a flight as it passes from fix to fix in the airspace. As the linear programming relaxations of these formulations are not very strong, branch and bound is needed to generate integral solutions. However, by developing a wide array of novel formulation strengthening techniques, the dependence on "pure" branch and bound, as well as the computation times, are actually reduced.
Helme (1994) has presented a method for the TFMP by designing a multicommodity minimum cost flow model over a network in space-time. To our knowledge, this method has not been fully tested, but it is expected that there will be severe dimensionality problems. To the best of our knowledge the TFMRP has not previously been addressed.

Contribution of This Work. We feel that our work makes the following contributions:

1. In the last fifteen years the field of polyhedral combinatorics has demonstrated that the key to solving large scale integer programming problems is to obtain strong formulations, which include facets of the convex hull of solutions. Our success in solving large scale, practical size instances of the TFMP lies exactly on this principle. We propose an integer programming model for the TFMP which is rather strong as some of the proposed inequalities are facet defining for the convex hull of solutions.

2. We address the complexity of the TFMP and show that it is NP-hard.

3. We illustrate how our models can be adjusted to account for several variations in the problem's characteristics, most notably how to handle banks in the hub and spoke system and how to reroute flights (the TFMRP problem).

4. When specialized for the MAGHP, we prove that the LP relaxation bound of our formulation is at least as strong when compared to all others proposed in the literature. As our model gives solutions that were almost always integral experimentally, there is no need for rounding heuristics that were used in Vranas et al. (1994a).

5. The solutions of the LP relaxation of the TFMP were almost always integral, so there was no need to branch and bound. In essense, our formulations reduce the problem to efficiently solving large scale linear programming problems. As a result, the computation times were reasonably small for large scale, realistic size problems involving thousands of flights. Short computational times and integrality properties are particularly important, since these models are intended to be used on-line and solved repeatedly during a day.

The paper is structured as follows. In Section 1 we formally introduce the TFMP and present our formulation. In Section 2 we address the complexity of the TFMP. In Section 3 we address modeling variations for the TFMP. In Section 4 we examine the theoretical properties of our formulation, proving that the proposed constraints are facet defining providing insights on the excellent computational performance. In Section 5 we report computational results and in Section 6 we include some concluding remarks and directions of future research. We include some technical proofs in the appendices.

\section{THE AIR TRAFFIC FLOW MANAGEMENT PROBLEM FORMULATION}

The National Airspace System (NAS) is divided into sectors. A map of the United States that displays all of the 


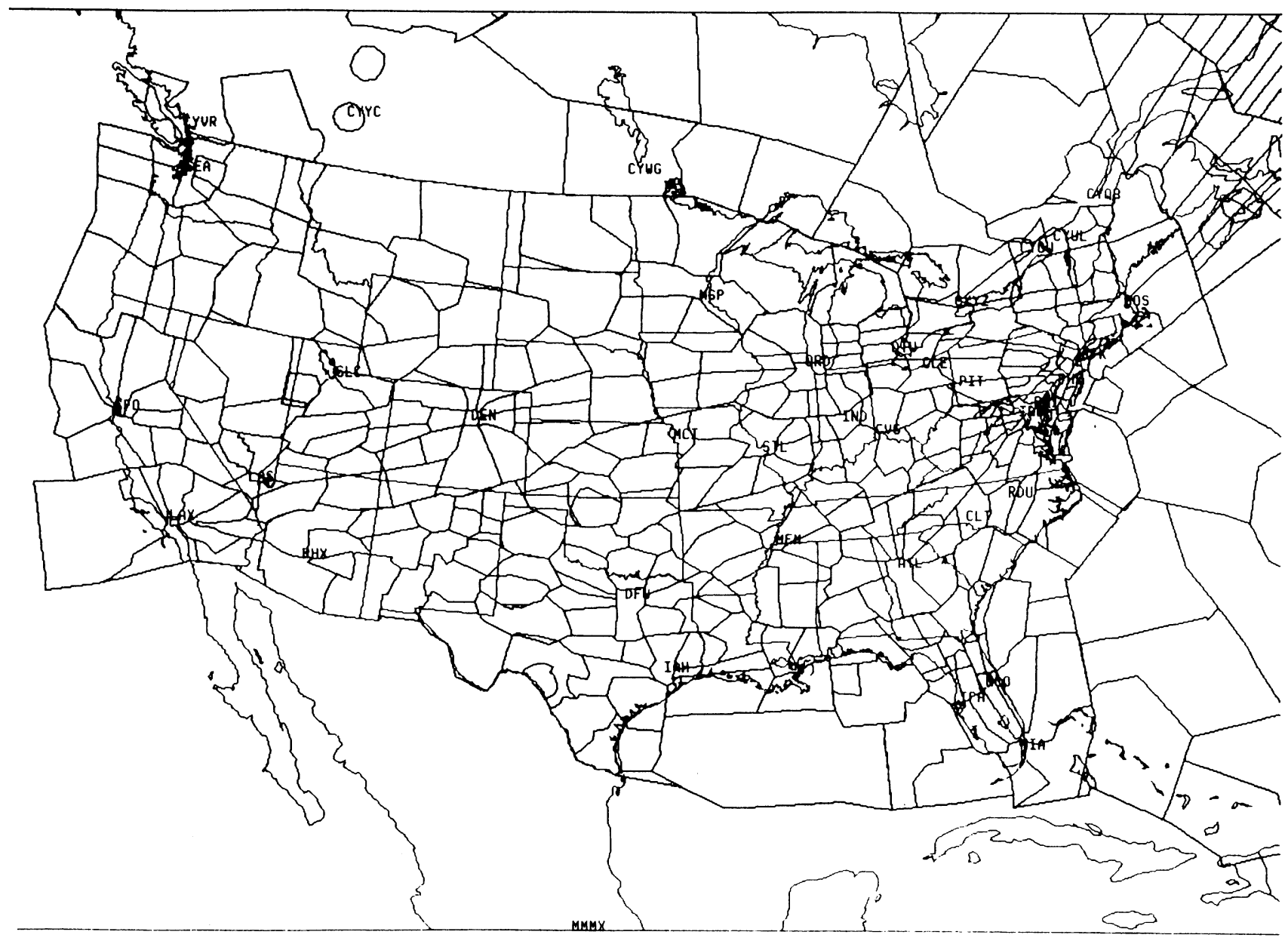

Figure 1. U.S. map with sector regions.

sector boundaries is given in Figure 1. Each flight passes through contiguous sectors while it is en route to its destination. There is a restriction on the number of airplanes that may fly within a sector at a given time. This number is dependent on the number of aircraft that an air traffic controller can manage at one time, the geographic location, and the weather conditions. We will refer to the restrictions on the number of aircraft in a given sector at a given time as the en route sector capacities. There are several key sectors throughout the United States that are often operated at full capacity. The issue of congestion at these sectors is as critical as congestion in the terminal areas, since the cost of holding an airborne aircraft is not dependent on the location of the aircraft. Thus, airborne delay costs could further be reduced if we could determine the optimal time for a flight to traverse the capacitated sectors. We first formulate the TFMP, examine the size of the formulation and make the connection with the groundholding problem.

\subsection{The 0-1 IP Formulation}

Consider a set of flights, $\mathscr{F}=\{1, \ldots, F\}$, a set of airports, $\mathscr{K}=\{1, \ldots, K\}$, a set of time periods, $\mathscr{T}=\{1, \ldots, T\}$, and a set of pairs of flights that are continued, $\mathscr{b}=$ $\left\{\left(f^{\prime}, f\right): f^{\prime}\right.$ is continued by flight $\left.f\right\}$. We shall refer to any particular time period $t$ as the "time $t$." The problem input data are given as follows:

Data.

$$
\begin{aligned}
N_{f}= & \text { number of sectors in flight } f \text { 's path, } \\
P(f, i)= & \left\{\begin{array}{c}
\text { the departure airport, if } i=1, \\
\text { the }(i-1)^{s t} \text { sector in flight } f \text { 's path, } \\
\text { if } 1<i<N_{f}, \\
\text { the arrival airport, if } i=N_{f},
\end{array}\right. \\
P_{f}= & \left(P(f, i): 1 \leqslant i \leqslant N_{f}\right), \\
D_{k}(t)= & \text { departure capacity of airport } k \text { at time } t, \\
A_{k}(t)= & \text { arrival capacity of airport } k \text { at time } t, \\
S_{j}(t)= & \text { capacity of sector } j \text { at time } t, \\
d_{f}= & \text { scheduled departure time of flight } f, \\
r_{f}= & \text { scheduled arrival time of flight } f, \\
s_{f}= & \text { turnaround time of an airplane after flight } f, \\
c_{f}^{g}= & \text { cost of holding flight } f \text { on the ground for one } \\
& \text { unit of time, } \\
c_{f}^{a}= & \text { cost of holding flight } f \text { in the air for one unit of } \\
& \text { time, } \\
l_{f j}= & \text { number of time units that flight } f \text { must spend } \\
& \text { in sector } j,
\end{aligned}
$$




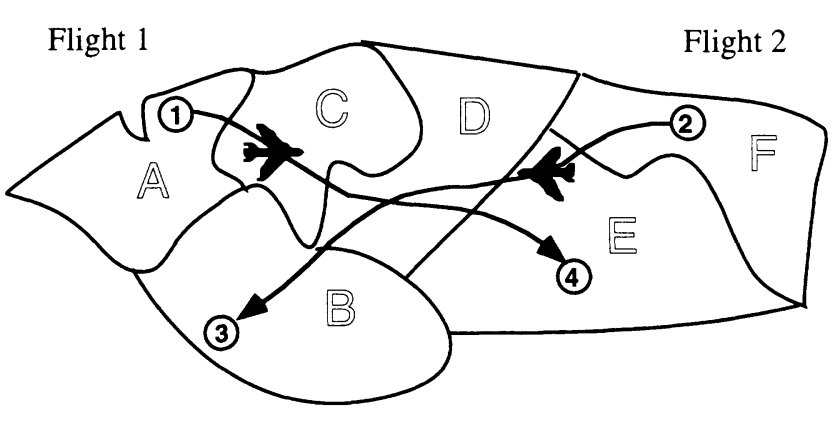

Figure 2. Two possible flight routes.

$T_{f}^{j}=$ set of feasible times for flight $f$ to arrive to sector $j=\left[\underline{T}_{f}^{j}, \bar{T}_{f}^{j}\right]$,

$\underline{T}_{f}^{j}=$ first time period in the set $T_{f}^{j}$, and

$\bar{T}_{f}^{j}=$ last time period in the set $T_{f}^{j}$.

Note that by "flight," we mean a "flight leg" between two airports. Also, flights referred to as "continued" are those flights whose aircraft is scheduled to perform a later flight within some time interval of its scheduled arrival.

Objective. The objective in the TFMP is to decide how much each flight is going to be held on the ground and in the air in order to minimize the total delay cost.

We model the problem as follows.

\section{Decision Variables.}

$w_{f t}^{j}= \begin{cases}1 & \text { if flight } f \text { arrives at sector } j \text { by time } t \\ 0 & \text { otherwise. }\end{cases}$

Note that the $w_{f t}^{j}$ are defined as being 1 if flight $f$ arrives at sector $j$ by time $t$. This definition using by and not at is critical to the understanding of the formulation. Also recall that we have also defined for each flight a list $P_{f}$ including the departure airport, the pertinent sectors and the arrival airport, so that the variable $w_{f t}^{j}$ will only be defined for those elements $j$ in the list $P_{f}$. Moreover, we have defined $T_{f}^{j}$ as the set of feasible times for flight $f$ to arrive to sector $j$, so that the variable $w_{f t}^{j}$ will only be defined for those times within $T_{f}^{j}$. Thus, in the formulation whenever the variable $w_{f t}^{j}$ is used, it is assumed that this is a feasible $(f, j, t)$ combination. Furthermore, one variable per flight-sector pair can be eliminated from the formulation by setting $w_{f, \bar{T}_{f}^{j}}^{j}=1$. Since flight $f$ has to arrive at sector $j$ by the last possible time in its time window, we can simply set it equal to one as a parameter before solving the problem. To ensure the clarity of the model, consider the following example which depicts two flights traversing a set of sectors. (See Figure 2.)

In this example, there are two flights, 1 and 2, each with the following associated data:

$P_{1}=(1, A, C, D, E, 4) \quad$ and

$P_{2}=(2, F, E, D, B, 3)$.

If we consider the current position of the aircraft to occur at time $t$, then the variables for these flights at this time will be: $w_{1, t}^{1}=1, w_{1, t}^{A}=1, w_{1, t}^{C}=1, w_{1, t}^{D}=0, w_{1, t}^{E}=0, w_{1, t}^{4}=0$,

and

$w_{2, t}^{2}=1, w_{2, t}^{F}=1, w_{2, t}^{E}=1, w_{2, t}^{D}=0, w_{2, t}^{B}=0, w_{2, t}^{3}=0$.

Having defined the variables $w_{f t}^{j}$ we can express several quantities of interest as linear functions of these variables as follows.

1. The variable $u_{f t}^{j}=1$ if flight $f$ arrives at sector $j$ at time $t$ and 0 otherwise, can be expressed as follows:

$u_{f t}^{j}=w_{f t}^{j}-w_{f, t-1}^{j}$ and vice versa, $w_{f t}^{j}=\sum_{t^{\prime} \leqslant t} u_{f t^{\prime}}^{j}$.

As expressed earlier, the variables $w_{f t}^{j}$ are only defined in the time range $T_{f}^{j}$, so that $w_{f,\left(T_{f}^{j}-1\right)}^{j}=0$. Furthermore, the constraint that a flight must arrive at sector $j$ at some time $t$, originally expressed by the restriction $\sum_{t \in \mathcal{T}_{f}^{j}} u_{f t}^{j}=1$ can now be replaced by the simpler expression $w_{f, \bar{T}_{f}^{j}}^{j}=1$. As previously mentioned, this can be handled as a parameter before the problem is solved, thus eliminating many variables and constraints. This substitution is fundamental to the performance of this model.

2. Noticing that the first sector for every flight represents the departing airport, the total number of time units that flight $f$ is held on the ground can be expressed as the actual departure time minus the scheduled departure time, i.e.,

$$
\begin{aligned}
g_{f} & =\sum_{t \in T_{f}^{k}, k=P(f, 1)} t u_{f t}^{k}-d_{f} \\
& =\sum_{t \in T_{f}^{k}, k=P(f, 1)} t\left(w_{f t}^{k}-w_{f, t-1}^{k}\right)-d_{f} .
\end{aligned}
$$

3. Noticing that the last sector for every flight represents the destination airport, the total number of time units that flight $f$ is held in the air can be expressed as the actual arrival time minus the scheduled arrival time minus the amount of time that the flight has been held on the ground, i.e.,

$$
\begin{aligned}
a_{f} & =\sum_{t \in T_{f}^{k}, k=P\left(f, N_{f}\right)} t u_{f t}^{k}-r_{f}-g_{f} \\
& =\sum_{t \in T_{f}^{k}, k=P\left(f, N_{f}\right)} t\left(w_{f t}^{k}-w_{f, t-1}^{k}\right)-r_{f}-g_{f} .
\end{aligned}
$$

The Objective Function. The objective of the formulation is to minimize total delay cost. Using the variables $g_{f}$ and $a_{f}$ for the amounts of ground and air delay respectively, as defined in items 2 and 3 above, the objective function can be expressed simply as follows:

$\operatorname{Min} \sum_{f \in \mathscr{F}}\left[c_{f}^{g} g_{f}+c_{f}^{a} a_{f}\right]$.

Substituting the expressions we derived in items 2 and 3 above for the variables $w_{f t}^{j}$, we obtain the following expression:

$$
\begin{aligned}
\operatorname{Min} \sum_{f \in \mathscr{F}}[ & c_{f}^{g}\left(\sum_{t \in T_{f}^{k}, k=P(f, 1)} t\left(w_{f t}^{k}-w_{f, t-1}^{k}\right)-d_{f}\right) \\
& +c_{f}^{a}\left(\sum_{t \in T_{f}^{k}, k=P\left(f, N_{f}\right)} t\left(w_{f t}^{k}-w_{f, t-1}^{k}\right)-r_{f}\right. \\
& \left.\left.\quad-\left(\sum_{t \in T_{f}^{k}, k=P(f, 1)} t\left(w_{f t}^{k}-w_{f, t-1}^{k}\right)-d_{f}\right)\right)\right] .
\end{aligned}
$$


Rearranging variables, we can now present the objective function along with the complete formulation.

$($ TFMP)

$$
\begin{aligned}
I Z_{T F M P}=\operatorname{Min} \sum_{f \in \mathscr{F}}[ & \left(c_{f}^{q}-c_{f}^{a}\right) \sum_{t \in T_{Y, k=P(f, 1)}^{k}} t\left(w_{f t}^{k}-w_{f, t-1}^{k}\right) \\
& +c_{f}^{a} \sum_{t \in T_{f, k}^{k}=P\left(f, N_{f}\right)} t\left(w_{f t}^{k}-w_{f, t-1}^{k}\right) \\
& \left.+\left(c_{f}^{a}-c_{f}^{q}\right) d_{f}-c_{f}^{a} r_{f}\right]
\end{aligned}
$$

subject to

$$
\begin{aligned}
& \sum_{f: P(f, 1)=k}\left(w_{f t}^{k}-w_{f, t-1}^{k}\right) \leqslant D_{k}(t) \quad \forall k \in \mathscr{K}, t \in \mathcal{T}, \\
& \sum_{f: P\left(f, N_{f}\right)=k}\left(w_{f t}^{k}-w_{f, t-1}^{k}\right) \leqslant A_{k}(t) \quad \forall k \in \mathscr{K}, t \in \mathcal{T},
\end{aligned}
$$

$$
\sum_{f: P(f, i)=j, P(f, i+1)=j^{\prime}, i<N_{f}}\left(w_{f t}^{j}-w_{f t}^{j^{\prime}}\right) \leqslant S_{j}(t) \quad \forall j \in \mathscr{F}, t \in \mathcal{T},
$$

$w_{f, t+l_{f}}^{j^{\prime}}-w_{f t}^{j} \leqslant 0 \quad\left\{\begin{aligned} \forall f & \in \mathscr{F}, t \in T_{f}^{j}, j=P(f, i), \\ j^{\prime} & =P(f, i+1), i<N_{f},\end{aligned}\right.$

$w_{f, t}^{k}-w_{f^{\prime}, t-s_{f}}^{k} \leqslant 0 \quad\left\{\begin{array}{c}\forall\left(f^{\prime}, f\right) \in \mathscr{b}, t \in T_{f}^{k}, \\ k=P(f, 1)=P\left(f^{\prime}, N_{f}\right),\end{array}\right.$

$w_{f, t}^{j}-w_{f, t-1}^{j} \geqslant 0 \quad \forall f \in \mathscr{F}, j \in P_{f}, t \in T_{f}^{j}$,

$w_{f t}^{j} \in\{0,1\} \quad \forall f \in \mathscr{F}, j \in P_{f}, t \in T_{f}^{j}$.

The first three constraints take into account the capacities of various aspects of the system. The first constraint ensures that the number of flights which may take off from airport $k$ at time $t$, will not exceed the departure capacity of airport $k$ at time $t$. Likewise, the second constraint ensures that the number of flights which may arrive at airport $k$ at time $t$, will not exceed the arrival capacity of airport $k$ at time $t$. In each case, the difference will be equal to one only when the first term is one and the second term is zero. Thus, the differences capture the time at which a flight uses a given airport. The third constraint ensures that the sum of all flights which may feasibly be in sector $j$ at time $t$ will not exceed the capacity of sector $j$ at time $t$. This difference gives the flights that are in sector $j$ at time $t$, since the first term will be 1 if flight $f$ has arrived in sector $j$ by time $t$ and the second term will be 1 if flight $f$ has arrived at the next sector by time $t$. So, the only flights that will contribute a value of 1 to this sum are those flights that have arrived at $j$ and have not yet departed from $j$ by time $t$.

Constraints (5) represent connectivity between sectors. They stipulate that if a flight arrives at sector $j^{\prime}$ by time $t+l_{f j}$, then it must have arrived at sector $j$ by time $t$ where $j$ and $j^{\prime}$ are contiguous sectors in flight $f$ 's path. In other words, a flight cannot enter the next sector on its path until it has spent $l_{f j}$ time units (the minimum possible) traveling through sector $j$, the current sector in its path.

Constraints (6) represent connectivity between airports. They handle the cases in which a flight is continued, i.e., the flight's aircraft is scheduled to perform a later flight within some time interval. We will call the first flight $f^{\prime}$ and the following flight $f$. Constraints (6) state that if flight $f$ departs from airport $k$ by time $t$, then flight $f^{\prime}$ must have arrived at airport $k$ by time $t-s_{f}$. The turnaround time, $s_{f}$, takes into account the time that is needed to clean, refuel, unload and load, and further prepare the aircraft for the next flight. In other words, flight $f$ cannot depart from airport $k$, until flight $f^{\prime}$ has arrived and spent at least $s_{f^{\prime}}$ time units at airport $k$.

Constraints (7) represent connectivity in time. Thus, if a flight has arrived by time $t$, then $w_{f t^{\prime}}^{j}$ has to have a value of 1 for all later time periods, $t^{\prime} \geqslant t$.

Important Remark. The major reason we used the variables $w_{f t}^{j}$, as opposed to the variables $u_{f t}^{j}$ is that the former variables nicely capture the three types of connectivity in TFMP: connectivity between sectors, connectivity between airports, and connectivity in time. Of course, given that the two sets of variables are linearly related, the same constraints can be captured using the $u_{f t}^{j}$ variables. We feel, however, that the variables $w_{f i}^{j}$ not only take connectivity naturally into account, but also they define connectivity constraints that are facets of the convex hull of solutions (see Section 3). As we report in Section 4, the LP relaxation of (TFMP) is almost always integral, i.e., the given formulation is a particularly strong one. We believe that the key for this is the use of the decision variables $w_{f i}^{j}$ in the formulation.

\subsection{Size of the Formulation}

Let $D$ be the maximum cardinality of the set of feasible times for flight $f$ to be in sector $j$ taken over all $f$ and $j$, i.e.,

$D=\max _{f \in \mathscr{F}, j \in P_{f}}\left|T_{f}^{j}\right|$.

Let

$X=\max _{f \in \mathscr{F}} N_{f}$,

be the maximum number of sectors that a flight passes through along its route, taken over all flights. Note that $X \geqslant$ 2 , since the departure and arrival airports are always counted as sectors on a flight's path. Let $|\mathscr{F}|$ be the total number of flights, $|\mathscr{T}|$ be the total number of time periods, $|\mathscr{K}|$ be the total number of airports, $|\nsubseteq|$ be the total number of sectors, and $|\mathscr{C}|$ be the total number of flights that are continued.

The actual number of variables $w_{f t}^{j}$ is $\Sigma_{f} \Sigma_{j \in P_{f}}\left|T_{f}\right|$ since each flight has a different number of sectors and number of feasible time intervals associated with it. An upper bound on the number of variables $w_{f t}^{j}$ will be

$|\mathscr{F}| D X$.

The exact number of constraints is

$$
\begin{aligned}
& 2|\mathscr{K} \| \mathscr{T}|+|\mathscr{F}||\mathscr{T}|+2 \sum_{f \in \mathscr{F}} \sum_{j \in P_{f}}\left|T_{f}^{j}\right| \\
& +\sum_{\substack{\left(f^{\prime}, f\right) \in \mathscr{C}, a=P(f, 1)=P\left(f^{\prime}, N_{f}\right)}} \min \left\{\left|T_{f}^{a}\right|,\left|T_{f^{\prime}}^{a}\right|\right\} .
\end{aligned}
$$


An upper bound on the number of constraints can then be calculated as

$2|\mathscr{T}||\mathscr{T}|+|\mathscr{F}||\mathscr{T}|+2|\mathscr{F}| D X+|\mathscr{G}| D$.

In order to get a feeling of the size of the formulation, let us consider an example that adequately represents the U.S. network:

1. $\mathscr{K}=20$ representing the most congested airports in the U.S.

2. $|\mathscr{T}|=14 * 12=168$, representing a 14 hour day with five-minute intervals.

3. $|\mathscr{F}|=200$, representing 200 sectors.

4. $|\mathscr{F}|=10000$, representing approximately half of the number of daily flights of major carriers.

5. $|\mathscr{C}|=8000$, representing an 80 -percent connectivity among flights.

6. $D=6$, representing an upper bound of half an hour that a flight can be late to any given sector.

7. $X=5$, representing an upper bound of at most five sectors in a flight's path.

For this example the number of variables is at most 300,000 and the number of constraints is at most 688,320 . The critical quantities that significantly affect the number of variables and constraints are $D, X$, and $|\mathscr{F}|$. If for example any of these parameters doubles, the number of variables doubles and the number of constraints nearly doubles.

\subsection{The Ground-Holding Problem as a Special Case}

As mentioned in the introduction, the ground-holding problem is a special case of the TFMP. If we remove the sector capacity constraints and the variables associated with the sectors, we obtain a new formulation of the MAGHP which, as we demonstrate in Section 5, leads to significant computational advantages compared to alternative formulations that have previously been proposed (see the introduction). Notice that $N_{f}=2$ for all $f \in \mathscr{F}$, since a flight's path consists solely of the departure and arrival airports.

Let us redefine the variables as:

$y_{f t}=w_{f t}^{k}$, for the departure airport, $k=P(f, 1)$.

$z_{f t}=w_{f t}^{k}$, for the arrival airport, $k=P(f, 2)$.

Also, let $T_{f}^{d}$ be the set of feasible departure times for flight $f$, and let $T_{f}^{a}$ be the set of feasible arrival times for flight $f$.

Using the new variables, the formulation (TFMP) specializes to the following new formulation of $(M A G H P)$ :

$(M A G H P)$

$$
\begin{aligned}
I Z_{M A G H P}=\operatorname{Min} \sum_{f \in F}[ & \left(c_{f}^{q}-c_{f}^{a}\right) \sum_{t \in T_{f}^{d}} t\left(y_{f t}-y_{f, t-1}\right) \\
& +c_{f}^{a} \sum_{t \in T_{f}^{q}} t\left(z_{f t}-z_{f, t-1}\right) \\
& \left.+\left(c_{f}^{a}-c_{f}^{q}\right) d_{f}-c_{f}^{a} r_{f}\right],
\end{aligned}
$$

subject to

$$
\begin{aligned}
\sum_{f: t \in T_{f}^{d}}\left(y_{f t}-y_{f, t-1}\right) & \leqslant D_{k}(t) \quad \forall k \in \mathscr{K}, t \in \mathscr{T}, \\
\sum_{f: t \in T_{f}^{q}}\left(z_{f t}-z_{f, t-1}\right) & \leqslant A_{k}(t) \quad \forall k \in \mathscr{K}, t \in \mathscr{T}, \\
z_{f, t}-y_{f, t-\left(r_{f}-d_{f}\right)} & \leqslant 0 \quad \forall f \in \mathscr{F}, t \in T_{f}^{a}, \\
y_{f, t}-z_{f^{\prime}, t-s_{f}} & \leqslant 0 \quad \forall\left(f^{\prime}, f\right) \in \mathscr{C}, t \in T_{f}^{d}, \\
y_{f, t}-y_{f, t-1} & \geqslant 0 \quad \forall f \in \mathscr{F}, t \in T_{f}^{d}, \\
z_{f, t}-z_{f, t-1} & \geqslant 0 \quad \forall f \in \mathscr{F}, t \in T_{f}^{a}, \\
y_{f t}, z_{f t} & \in\{0,1\} \quad \forall f \in \mathscr{F}, t \in \mathscr{T} .
\end{aligned}
$$

The first two constraints incorporate the capacity restrictions of the departure and arrival airports. The next constraint is the sector connectivity constraint, which is equivalent to constraint (5) in the TFMP formulation. However, for the ground-holding problem the only elements in the path are the departure airport and the arrival airport. So this constraint connects these two elements by making sure that flight $f$ cannot arrive at time $t$ unless it has departed by at least $t$ minus the minimum flight time. The next constraint is the flight connectivity constraint, which is equivalent to constraint (6) in the TFMP formulation. The last two constraints are time connectivity constraints, which are equivalent to constraint (7) in the formulation (TFMP).

Using the previous definitions, an upper bound on the number of variables is $2|\mathscr{F}| D$, and an upper bound on the number of constraints is $2|\mathscr{H}||\mathscr{T}|+3|\mathscr{F}| D+|\mathscr{C}| D$. For the same example as in the end of the previous subsection, an upper bound on the number of variables in the above formulation is 120,000 and an upper bound on the number of constraints is 234,720 .

If we remove the constraint (12) and consider the set $\mathscr{K}$ to be the singleton set, then we have a valid formulation for SAGHP, which we will call $(S A G H P)$. We define the feasible regions for the formulations (TFMP), $(M A G H P)$, and $(S A G H P)$ as $I P_{T F M P}, I P_{M A G H P}$, and $I P_{S A G H P}$, respectively.

The variables used in the formulation in Vranas et al. (1994a) are defined differently: $u_{f t}=1$ if flight $f$ takes off at time $t$ and $v_{f t}=1$ if flight $f$ arrives at time $t$. These are linearly related to variables $y_{f t}$ and $z_{f t}$ as per the relationship given by (1). As already mentioned, the groundholding delays can be expressed in terms of these variables in the following manner:

$g_{f}=\sum_{t \in T_{f}^{d}} t u_{f t}-d_{f}$,

as can the airholding delay,

$a_{f}=\sum_{t \in T_{f}^{g}} t v_{f t}-r_{f}-g_{f}$.

In Vranas et al. (1994a), it is assumed that when the departure capacity is large, without loss of generality, $a_{f}=0$, thus implying that all of the delay would be taken on the 
ground before departure. This gives an equivalent expression for $g_{f}$ as, $g_{f}=\sum_{t \in T_{f}^{a}} t v_{f t}-r_{f}$, which contains no departure information, thus eliminating the variables $u_{f t}$ from the formulation. Moreover, instead of the flight connectivity constraints (12), the following constraints,

$g_{f^{\prime}}-\left(d_{f}-s_{f^{\prime}}-r_{f^{\prime}}\right) \leqslant g_{f}$,

establish connectivity between the arriving flight $f^{\prime}$ and the departing flight $f$ by forcing the amount of ground-hold for flight $f$ to be at least the amount that flight $f^{\prime}$ arrives late, $g_{f^{\prime}}$, minus the amount of slack time, $d_{f}-s_{f^{\prime}}-r_{f}$. The description of the feasible space in Vranas et al. (1994a) expressed in the $z_{f t}$ space as per the relationship (1) is as follows:

$$
\begin{aligned}
I P_{V B O}=\left\{z_{f t} \in\{0,1\} \mid \sum_{f}\left(z_{f t}-z_{f, t-1}\right) \leqslant A_{k}(t),\right. & \\
& \sum_{t \in T_{f}^{a}}\left(z_{f t}-z_{f, t-1}\right)=1, \\
& g_{f}=\sum_{t \in T_{f}^{a}} t\left(z_{f t}-z_{f, t-1}\right)-r_{f}, \\
& \left.g_{f^{\prime}}-\left(d_{f}-s_{f^{\prime}}-r_{f^{\prime}}\right) \leqslant g_{f}, z_{f t}-z_{f, t-1} \geqslant 0\right\} .
\end{aligned}
$$

Terrab and Paulose (1993) use the same variables, $v_{f t}$ as in Vranas et al. (1994a). However, they express the flight connectivity constraints as follows:

$$
\sum_{t \in T_{f}^{a}, t \leqslant \tau} v_{f t}-\underset{t^{\prime} \in T_{f}^{a}, t^{\prime} \leqslant \tau-s_{f}-\left(r_{f}-d_{f}\right)}{ } v_{f^{\prime} t^{\prime}} \leqslant 0 .
$$

Constraint (18) forces connectivity, since if the second sum is zero then flight $f^{\prime}$ has not landed by time $\tau-s_{f^{\prime}}-\left(r_{f}-\right.$ $d_{f}$ ), which is time period $\tau$ minus the turnaround time, minus the flight time of $f$. This forces the first sum to be zero so that flight $f$ can not land before time $\tau$. The description of their formulation expressed in the $z_{f t}$ space as per the relationship (1) is:

$$
\begin{gathered}
I P_{T P}=\left\{z_{f t} \in\{0,1\} \mid \sum_{f}\left(z_{f t}-z_{f, t-1}\right) \leqslant A_{k}(t),\right. \\
\sum_{t \in T_{f}^{a}}\left(z_{f t}-z_{f, t-1}\right)=1, \sum_{t \in T_{f}^{a}, t \leqslant \tau}\left(z_{f t}-z_{f, t-1}\right) \\
-\sum_{t^{\prime} \in T_{f}^{a}, t^{\prime} \leqslant \tau-s_{f}}-\left(r_{f}-d_{f}\right) \\
\left.z_{f t}-z_{f, t-1} \geqslant 0\right\} .
\end{gathered}
$$

If we specialize our formulation for the case of large departure capacities and use only the variables, $z_{f t}\left(y_{f t}=\right.$ $\left.z_{f, t+\left(r_{f}-d_{f}\right)}\right)$, we obtain:

$$
\begin{aligned}
& I P_{M A G H P}^{\prime}=\left\{z_{f t} \in\{0,1\} \mid \sum_{f: t \in T_{f}^{a}}\left(z_{f t}-z_{f, t-1}\right) \leqslant A_{k}(t),\right. \\
& \sum_{t \in T_{f}^{a}}\left(z_{f t}-z_{f, t-1}\right)=1 \\
& z_{f, t+\left(r_{f}-d_{f}\right)}-z_{f^{\prime}, t-s_{f}} \leqslant 0 \\
&\left.z_{f, t}-z_{f, t-1} \geqslant 0\right\} .
\end{aligned}
$$

In all of these formulations, the expression $\sum_{t \in T_{f}^{a}}\left(z_{f t}\right.$ $\left.z_{f, t-1}\right)=1$ reduces to the expression $z_{f, \bar{T}_{f}}=1$. This telescoping property is due to the unique definition of the decision variables as flights arriving by some time $t$ rather than at time $t$.

If we denote the polyhedra corresponding to the linear programming relaxations of $I P_{M A G H P}^{\prime}, I P_{V B O}$, and $I P_{T P}$ as $P_{M A G H P}^{\prime}, P_{V B O}$, and $P_{T P}$ and denote their corresponding values as $Z_{M A G H P}^{\prime}, Z_{V B O}$, and $Z_{T P}$, then we can state the following proposition whose proof is included in Appendix A.

Proposition 1. $I P_{T P}=I P_{V B O}=I P_{M A G H P}^{\prime} \subseteq P_{M A G H P}^{\prime} \subseteq$ $P_{T P} \subseteq P_{V B O}$, and correspondingly, $Z_{V B O} \leqslant Z_{T P} \leqslant Z_{M A G H P}^{\prime}$ $\leqslant I Z_{M A G H P}^{\prime}=I Z_{V B O}=I Z_{T P}$

Therefore, the LP relaxation of $(M A G H P)$ gives bounds that are at least as strong as those from the LP relaxations of either Vranas et al. (1994a) or Terrab and Paulose (1993).

\section{COMPLEXITY OF THE TFMP}

In this section we show that the TFMP is an NP-hard problem.

Theorem 1. The TFMP with all capacities equal to 1 is NP-hard.

Proof. We show that job-shop scheduling (see Garey and Johnson 1979) reduces to TFMP.

\section{JOB SHOP SCHEDULING PROBLEM (JSP)}

INSTANCE: Number $m \in Z^{+}$of processors, set $J$ of jobs, each $j \in J$ consisting of an ordered collection of tasks $t_{k}[j]$, $1 \leqslant k \leqslant n_{j}$, for each task $t$ a length $l(t) \in Z_{0}^{+}$and a processor $p(t) \in\{1,2, \ldots, m\}$, where $p\left(t_{k}[j]\right) \neq$ $p\left(t_{k+1}[j]\right)$ for all $j \in J$ and $1 \leqslant k<n_{j}$, and a deadline $D \in$ $Z^{+}$.

QUESTION: Is there a job-shop schedule for $J$ that meets the overall deadline, i.e., a collection of one-processor schedules $\sigma_{i}$ mapping $\{t: p(t)=i\}$ into $Z_{0}^{+}, 1 \leqslant i \leqslant m$, such that $\sigma_{i}(t)>\sigma_{i}\left(t^{\prime}\right)$ implies $\sigma_{i}(t) \geqslant \sigma_{i}\left(t^{\prime}\right)+l(t)$, such that $\sigma_{i^{\prime}}\left(t_{k+1}[j]\right) \geqslant \sigma_{i}\left(t_{k}[j]\right)+l\left(t_{k}[j]\right)$ where $i^{\prime}=p\left(t_{k+1}[j]\right)$ and $i=p\left(t_{k}[j]\right)$, for all $j \in J$ and $1 \leqslant k \leqslant n_{j}$, and such that for all $j \in J, \sigma_{i}\left(t_{n_{j}}[j]\right)+l\left(t_{n_{j}}[j]\right) \leqslant D$ where $i=p\left(t_{n_{j}}[j]\right)$ ?

For each job we create an aircraft. For each processor we associate an airport or sector. Task $t_{k}[j]$ of job $j$ corresponds to a flight segment, $f_{k}[j]$ of aircraft $j$. Given a collection of tasks, $t_{k}[j]$ of job $j$, we associate a list of airports and sectors to be visited by aircraft $j$. Furthermore, the processing time of task $t_{k}[j]$ corresponds to the time required to perform the flight segment, $f_{k}[j]$. We obtain a list of airports and sectors, $\left(A_{j}^{1}, S_{j}^{2}, \ldots, A_{j}^{k}\right.$, $\left.S_{j}^{(k+1)}, \ldots, A_{j}^{n_{j}}\right)$, and a list of the flight segment times, $\left(t_{A_{j}}^{1}\right.$, $\left.t_{S_{j}}^{2}, \ldots, t_{A_{j}}^{k}, t_{S_{j}}^{k+1}, \ldots, t_{A_{j}}^{n_{j}}\right)$, for each aircraft $j$ by the relationships: 
$A_{j}^{1}=p\left(t_{j}[1]\right), \quad t_{A_{j}}^{1}=l\left(t_{j}[1]\right)$

$S_{j}^{2}=p\left(t_{j}[2]\right), \quad t_{S_{j}}^{2}=l\left(t_{j}[2]\right)$

$S_{j}^{3}=p\left(t_{j}[3]\right), \quad t_{S_{j}}^{3}=l\left(t_{j}[3]\right)$

$A_{j}^{n_{j}}=p\left(t_{j}\left[n_{j}\right]\right), \quad t_{A_{j}}^{n_{j}}=l\left(t_{j}\left[n_{j}\right]\right)$.

So by finding a job-shop schedule that satisfies the given conditions, we will find a solution to the transformed problem such that all flights are performed by the deadline $D$. Also, according to the relationship $\sigma_{i^{\prime}}\left(t_{k+1}[j]\right) \geqslant \sigma_{i}\left(t_{k}[j]\right)+$ $l\left(t_{k}[j]\right)$ where $i^{\prime}=p\left(t_{k+1}[j]\right)$ and $i=p\left(t_{k}[j]\right)$, no two tasks will ever performed simultaneously on the same processor, which is equivalent to limiting the capacities of airports and sectors to one. Moreover, the relationship, $\sigma_{i}(t)>\sigma_{i}\left(t^{\prime}\right)$ implies $\sigma_{i}(t)$ $\geqslant \sigma_{i}\left(t^{\prime}\right)+l(t)$, dictates that a task can not be processed unless the previous task has completed. This stipulation guarantees connectivity between flights, and sectors, as specified by the set of tasks for each aircraft. Thus, all the constraints of the TFMP will be satisfied if and only if there exists a feasible job-shop schedule.

\section{MODELING VARIATIONS}

Our goal in this section is to demonstrate that the formulation (TFMP) can be easily extended in many directions to take into account several variations of the model.

\subsection{Dependence Between Arrival and Departure Capacities}

The interdependence between the arrival and departure capacities of airports results from the fact that the same runways are used for both arrivals and departures. Thus, the runway allocation will determine how an airport's available capacity is allocated between the arrivals and departures at a given time. By operating under a specific runway configuration, arrival and departure capacities can be adjusted. This will significantly influence airport efficiency. By choosing a particular configuration of runways for a given time, the capacity allocation will be fixed. The complete set of runways for Logan Airport is given in Figure 3. A common configuration used at Logan Airport is to use runways $4 \mathrm{~L}$ and $4 \mathrm{R}$ for arriving flights and to use runways 9 and $4 R$ for departing flights. Notice that since runway $4 R$ is the longest runway and certain types of aircraft require a long runway, it is used for both arrivals and departures. Since it takes longer for an aircraft to arrive than to depart, if all the capacity at Logan Airport is allocated to arrivals then 52 flights could arrive, and if all the capacity is allocated to departures then 62 flights could depart within an hour. We review briefly ideas introduced in Gilbo (1993) and Vranas et al. (1994a). We represent the runway allocation by a set of linear constraints indexed by $i$ for airport $k$ at time $t$ of the type

$\alpha_{k t}^{i} D_{k}(t)+\beta_{k t}^{i} A_{k}(t) \leqslant \gamma_{k t}^{i} \quad \forall k \in \mathscr{K}, t \in \mathcal{T}, i \in I_{k t}$,

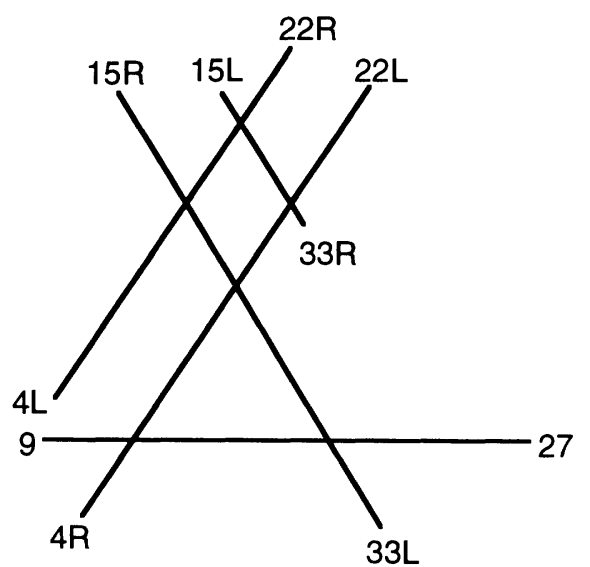

Figure 3. Complete runway configuration for Logan Airport.

where $\alpha_{k t}^{i}, \beta_{k t}^{i}$, and $\gamma_{k t}^{i}$ are given constants. The region formed by the above constraints gives a complete depiction of all the possible runway allocations at a given time, and likewise, all possible departure and arrival capacity assignments. So, for the Logan example, the set of linear constraints is given in Figure 4.

In order to solve this variation, we treat $D_{k}(t)$ and $A_{k}(t)$ as variables that satisfy constraints (19) and add them to (TFMP). We can further reduce the size of the resulting formulation by eliminating the variables $D_{k}(t)$ and $A_{k}(t)$ by incorporating constraints (2) and (3) taken at equality into (19) as follows:

$$
\begin{aligned}
& \alpha_{k t}^{i} \sum_{f: t \in T_{f}^{k}, k=P(f, 1)}\left(w_{f t}^{k}-w_{f, t-1}^{k}\right) \\
& \quad+\beta_{k t}^{i} \sum_{f: t \in T_{f, k=P\left(f, N_{f}\right)}^{k}}\left(w_{f t}^{k}-w_{f, t-1}^{k}\right) \leqslant \gamma_{k t}^{i} .
\end{aligned}
$$

The addition of this constraint to (TFMP) incorporates the dependence between the arrival and departure capacity assignments without the addition of any new variables.

\subsection{Hub Connectivity with Multiple Connections}

Given that many airlines now control key hub airports through which most of their flights are directed, it is no

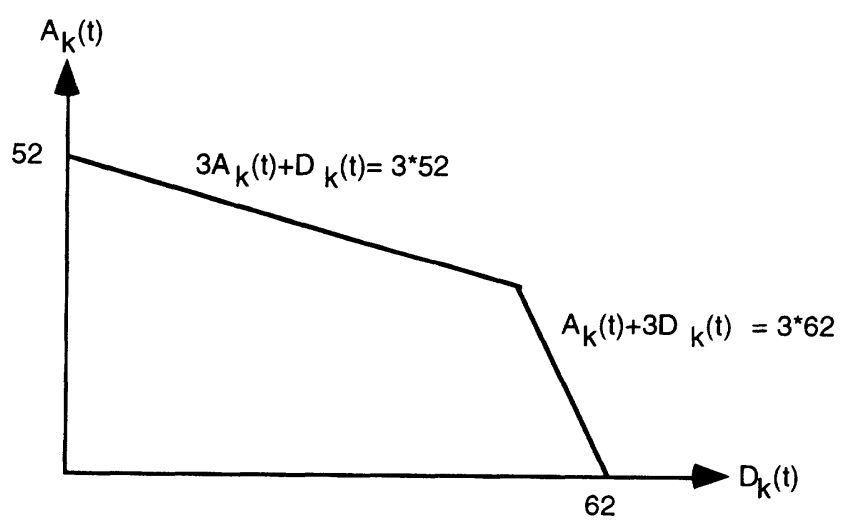

Figure 4. Runway departure/arrival allocation for the specific configuration. 
longer obvious which aircraft will fly a subsequent flight. At these hubs, many airplanes are capable of performing any one of multiple consecutive flights. We refer to the issue of assigning aircraft to continuing flights as hub connectivity. This can be achieved by extending the model as follows. For each arriving flight $f^{\prime}$ that is continued there is a set of flights $R_{f^{\prime}}$ that can continue flight $f^{\prime}$. Introducing the $0-1$ variables $x_{f^{\prime} f}$, which take on the value 1 if flight $f^{\prime}$ is continued by flight $f \in R_{f^{\prime}}$ and 0 otherwise, we alter constraint (6) as follows

$$
\begin{aligned}
& w_{f t}^{k}-w_{f^{\prime}, t-s_{f}}^{k} \leqslant 1-x_{f^{\prime} f} \\
& \forall\left(f^{\prime}, f\right) \in \mathscr{C}, t \in T_{f}^{k}, k=P(f, 1)=P\left(f^{\prime}, N_{f^{\prime}}\right),
\end{aligned}
$$

and add the constraint that each continued flight $f^{\prime}$ has to be assigned to a flight in $R_{f^{\prime}}$ :

$$
\sum_{f \in R_{f}^{\prime}} x_{f^{\prime} f}=1 \text {. }
$$

\subsection{Banks of Flights}

With the evolution of the hub and spoke system, airlines have a set of flights (banks) that are scheduled to arrive at a hub airport and another set scheduled to depart within a small time window of the arrival bank. Each arriving aircraft will be assigned to perform at most one of the departing flights. This situation is similar to hub connectivity, except that airlines seek to minimize the time between the departure of the first and the last flight in the bank. Let $B$ be the set of flights in a bank. We define the decision variables

$y_{B, t}= \begin{cases}1 & \text { if the first flight } f \text { in } B \text { arrives by time } t, \\ 0 & \text { otherwise. }\end{cases}$

$z_{B, t}= \begin{cases}1 & \text { if the last flight } f \text { in } B \text { arrives by time } t \\ 0 & \text { otherwise. }\end{cases}$

These definitions require the constraints:

$$
\begin{array}{ll}
y_{B, t}-w_{f, t}^{k} \geqslant 0 & \forall f \in B, t \in T_{f}^{k}, k=P\left(f, N_{f}\right), \\
z_{B, t}-w_{f, t}^{k} \leqslant 0 & \forall f \in B, t \in T_{f}^{k}, k=P\left(f, N_{f}\right) .
\end{array}
$$

We also need the additional time connectivity constraints for these variables

$$
\begin{aligned}
& y_{B, t}-y_{B, t-1} \geqslant 0 \quad \forall t \in \mathscr{T}, \\
& z_{B, t}-z_{B, t-1} \geqslant 0 \quad \forall t \in \mathscr{T} .
\end{aligned}
$$

The objective function of minimizing the "spread" in the arrival times for the flights in the bank $B$ can be modeled as follows:

$$
\min \sum_{t \in \mathscr{T}} t\left(z_{B, t}-z_{B, t-1}\right)-\sum_{t \in \mathscr{T}} t\left(y_{B, t}-y_{B, t-1}\right) .
$$

This is equivalent to determining

$$
\begin{aligned}
& \min \sum_{t \in \mathcal{T}}\left(\max _{f \in B, k=P\left(f, N_{f}\right)} t\left(w_{f t}^{k}-w_{f, t-1}^{k}\right)\right. \\
&\left.-\min _{f \in B, k=P\left(f, N_{f}\right)} t\left(w_{f t}^{k}-w_{f, t-1}^{k}\right)\right) .
\end{aligned}
$$

With the addition of these new variables, new constraints, and new objective function, banking can be incorporated into the formulation. An alternative approach to handle banking constraints is proposed in Ball (1993).

\subsection{Rerouting of Aircraft}

Very often, extreme weather conditions force the capacities of some sectors (and airports) in the NAS to drop significantly or even to become zero. Air traffic controllers are then forced to use alternative routes for aircraft passing through these sectors to accommodate these changes in capacities (see Figure 5 for an example). Currently, these rerouting decisions are handled through the experience of the air traffic controllers and not through a formal optimization model. We illustrate in this section that our models can be extended to efficiently accommodate dynamic rerouting decisions. We present two possible approaches: the path approach and the sector approach.

The path approach first defines $Q_{f}$ as a set of possible routes that flight $f$ may fly. In the formulation (TFMP) we have assumed that $Q_{f}$ only contains one route, which we have denoted as $P_{f}$. In order for the formulation to be of manageable (but still large) size we need to restrict the size of $Q_{f}$. We extend the TFMP variables in the following manner:

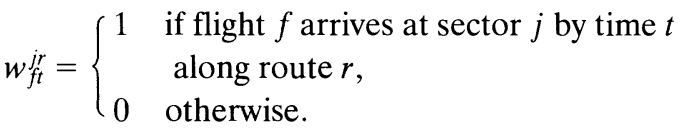

Clearly, the variables $w_{f t}^{j}$ defined in Section 1 can be written as:

$w_{f t}^{j}=\sum_{r \in Q_{f}} w_{f t}^{j r}$.

Moreover, since the departure and arrival airports will remain the same for a given flight over all routes, $P(f, 1)$ and $P\left(f, N_{f}\right)$ will be independent of the particular route. Using the newly defined variables we can modify the TFMP to include rerouting. The size of the resulting formulation will be at most a factor $\max _{f}\left|Q_{f}\right|$ larger than the TFMP formulation. This implies that we should be able to handle problems with a relatively small number of alternative paths.

The sector approach decides at each sector in its route which sector to enter next. We need to define $N(f, j)$, the set of sectors that flight $f$ can enter immediately after exiting sector $j$, as well as $P(f, j)$, the set of sectors that flight $f$ can enter immediately before entering sector $j$. We extend the TFMP variables in the following manner:

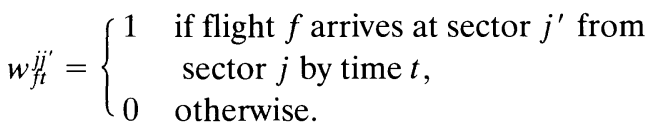

Clearly, the variables $w_{f t}^{j}$ defined in Section 1 can be written as:

$w_{f t}^{j}=\sum_{j^{\prime} \in N(f, j)} w_{f t}^{j^{\prime} j}$. 


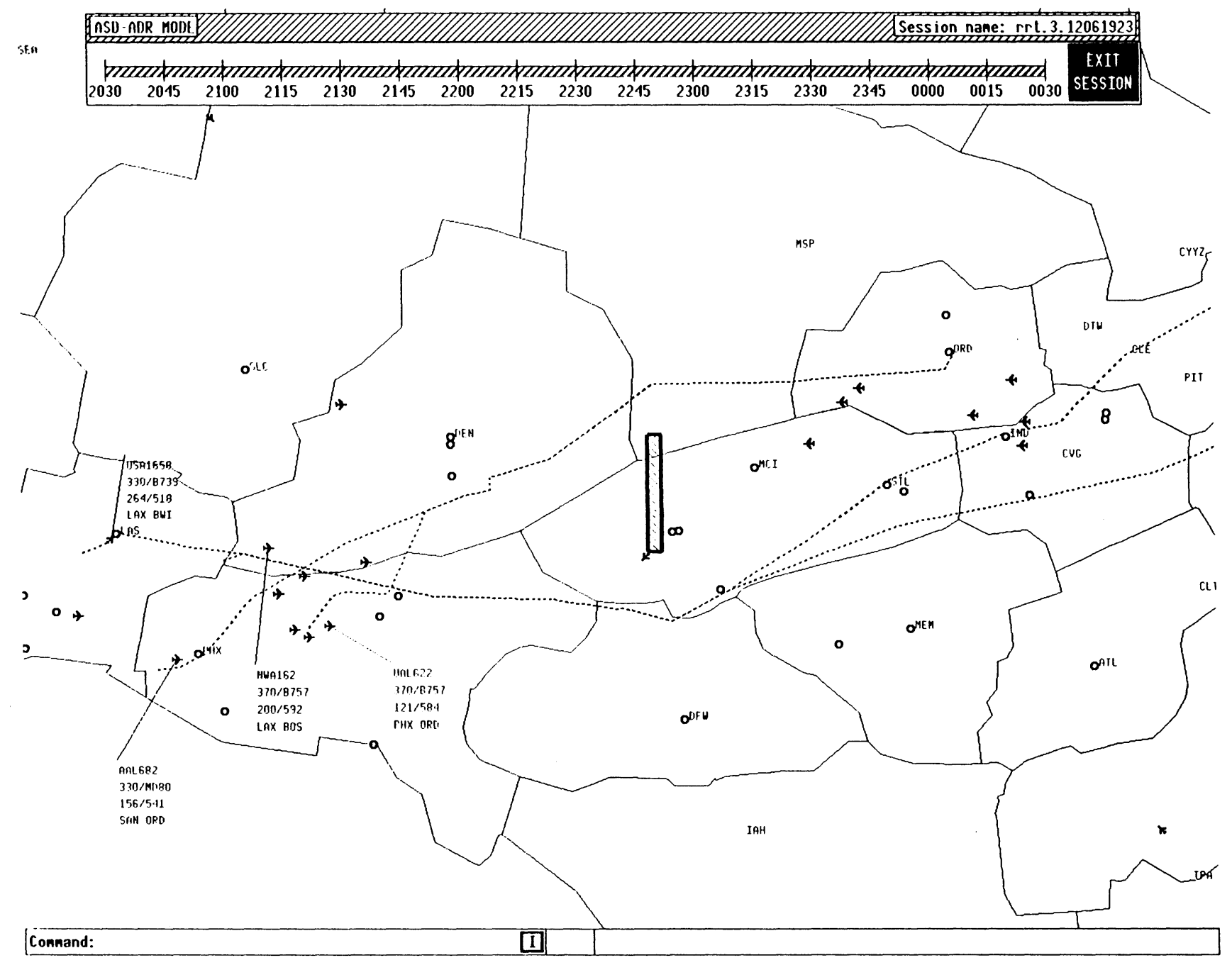

Figure 5. Alternative routes taken as flights avoid a low capacity region.

As before, the departure and arrival airports will remain the same for a given flight over all routes, $P(f, 1)$ and $P(f$, $N_{f}$ ) will be independent of the particular choice of sectors.

Although both the path and sector approach will give correct formulations for the TFMRP, further investigation is needed to determine which variable definition will perform the best in terms of integrality and computation speed.

\section{INSIGHTS FROM THE POLYHEDRAL STRUCTURE}

In Section 5 we report computational results for the TFMP based on the formulation (TFMP). Even for large scale problems and for a variety of problem parameters, the solutions of the LP relaxation of both (TFMP) and $(M A G H P)$ were integral. In the tradition of polyhedral combinatorics in mathematical programming, we examine the polyhedral structure of $P_{T F M P}$ and $P_{M A G H P}$ in order to obtain a deeper understanding of why this formulation performs so well computationally. Given a set $S$ we denote with $\operatorname{conv}(S)$ the convex hull of solutions in $S$. In particular we will now address the following questions:
1. Are the polyhedra $P_{T F M P}$ and $P_{M A G H P}$ integral? If not, is the optimal solution to the optimization problem integral if we impose the simplification that $c_{g}=c_{f}^{g}$ and $c_{a}$ $=c_{f}^{a}$ for all $f \in \mathscr{F}$ ?

2. Are the constraints in (TFMP) and (MAGHP) facets of $\operatorname{conv}\left(I P_{T F M P}\right)$ and $\operatorname{conv}\left(I P_{M A G H P}\right)$ respectively?

We summarize our findings in the following theorem.

Theorem 2. 1. The polyhedra $P_{T F M P}$ and $P_{M A G H P}$ are not integral. Even with the simplification that $c_{g}=c_{f}^{g}$ and $c_{a}=$ $c_{f}^{a}$ for all $f \in \mathscr{F}$, integral solutions are not obtained.

2. Inequalities (11), (12), (13), and (14) are facets for $\operatorname{conv}\left(I P_{M A G H P}\right)$, while the constraints (9) and (10) are not. Inequalities (5), (6), and (7) are facets for conv $\left(I P_{T F M P}\right)$, while the constraints (2), (3), and (4) are not.

As the proofs of the theorem are somewhat technical, we have placed them in Appendices B, and C, respectively.

The previous theorem gives some partial insight on the usefulness of the new variables we introduced, which make it easy to express sharply the various types of connectivity in the problem. While the formulations are not integral, 
Table I

Results at the Infeasibility Border for 1,000 Flights

\begin{tabular}{cccccc}
\hline$|\mathscr{F}|$ & $|\mathscr{G}| /|\mathscr{F}|$ & $\begin{array}{c}\text { Dep. } \\
\text { Capacity }\end{array}$ & $\begin{array}{c}\text { Arr. } \\
\text { Capacity }\end{array}$ & Time & $\begin{array}{c}\% \\
\text { Nonint. }\end{array}$ \\
\hline 1,000 & 0.20 & 32 & 15 & 262 & 0 \\
1,000 & 0.40 & 17 & 10 & 741 & 4 \\
1,000 & 0.60 & 20 & 14 & 359 & 0 \\
1,000 & 0.80 & 20 & 20 & 283 & 0 \\
\hline
\end{tabular}

the inequalities that the three types of connectivity impose are indeed facets. As the solutions obtained were integral for a wide spectrum of examples and parameters, we did not investigate further the determination of other facets.

\section{INSIGHTS FROM COMPUTATIONS}

In this section we report the results of a series of computational experiments that we conducted. In performing the computational experiments, we aimed to address the following questions.

1. How frequently are the solutions obtained by solving the LP relaxations of (TFMP) and (MAGHP) integral?

2. How is the integrality of solutions affected by the various problem parameters and the size of the problem?

3. How is the computational time required to obtain an optimal solution affected by the various problem parameters and the size of the problem?

4. How does the present approach compare with other approaches in the literature?

5. Given that the TFMP needs to be solved on line for controlling air traffic in the United States, perhaps the most important question to ask is: How large problems can we solve in reasonable computational times? In other words, is the present approach a realistic method to control air traffic in the United States?

Ground-Holding Problem Test Cases. We performed computational experiments on datasets used in Vranas et al. (1994a) on the Ground-Holding Problem. Specifically, we looked at the datasets consisting of two and six airports with 500 flights per airport, 1000 and 3000 flights, respectively. Some adjustments in the data were necessary in order to accommodate the differences between the two models. In particular, the previous model did not include of any departure data, as all of the optimization was done with respect to arrivals. Thus, we generated departure data (times and capacities) that were compatible with the existing arrival data.

\section{Table II}

Results at the Infeasibility Border for 3,000 Flights

\begin{tabular}{cccccc}
\hline$|\mathscr{F}|$ & $|\mathscr{C}| /|\mathscr{F}|$ & $\begin{array}{c}\text { Dep. } \\
\text { Capacity }\end{array}$ & $\begin{array}{c}\text { Arr. } \\
\text { Capacity }\end{array}$ & Time & $\begin{array}{c}\% \\
\text { Nonint. }\end{array}$ \\
\hline 3,000 & 0.20 & 20 & 20 & 5,475 & 0 \\
3,000 & 0.40 & 20 & 20 & 4,703 & 0 \\
3,000 & 0.60 & 20 & 20 & 5,407 & 0 \\
3,000 & 0.80 & 20 & 20 & 9,411 & 0 \\
\hline
\end{tabular}

Table III

Previous Results at the Infeasibility Border for 1,000 Flights

\begin{tabular}{cccccrc}
\hline$|\mathscr{F}|$ & $|\mathscr{C}| /|\mathscr{F}|$ & $\begin{array}{c}\text { Dep. } \\
\text { Capacity }\end{array}$ & $\begin{array}{c}\text { Arr. } \\
\text { Capacity }\end{array}$ & $\begin{array}{c}\text { LP } \\
\text { Time }\end{array}$ & $\begin{array}{r}\text { Total } \\
\text { Time }\end{array}$ & $\begin{array}{c}\% \\
\text { Nonint. }\end{array}$ \\
\hline 1,000 & 0.20 & $\infty$ & $(12,14)$ & 258 & 374 & 6.3 \\
1,000 & 0.40 & $\infty$ & 10 & 327 & 894 & 8.4 \\
1,000 & 0.60 & $\infty$ & 11 & 377 & 6,958 & 12.8 \\
1,000 & 0.80 & $\infty$ & 10 & 453 & 9,512 & 16.8 \\
\hline
\end{tabular}

As in Vranas et al. (1994a), for each of these cases, four levels of flight connectivity were considered. These levels give the ratios of continued flight to total flights, $|\mathscr{6}| /|\mathscr{F}|$, as $0.20,0.40,0.60$, and 0.80 .

We considered 15-minute time intervals taken over a 16-hour day. All experiments were performed on a Sun SPARCstation 10 model 41. GAMS was used as the modeling tool and CPLEXMIP 2.1 was used as the solver. The results that we obtained using the above datasets and our $(M A G H P)$ formulation are summarized in Tables I and II. Tables I and II give results at the infeasibility border for each case. The infeasibility border is the set of critical values for the departure and arrival capacities, in units of flights per time interval, under which the problem becomes infeasible. We expect that it is in this region that the problem is very relevant practically and is harder to solve. The critical capacity levels were found by a series of trial and error tests. The times reported are in CPU seconds and the \% Nonint column is the percentage of total flights whose solution was noninteger. If we compare these results with the results from Vranas et al. (1994a) (see Tables III and IV), we can see that the largest amount of improvement occurred in the integrality of the solutions. The computational times for solving our LP for 1000 flights (see Table I; column Time) are comparable to the time required to solve their LP (see Table III; column LP Time), while for the 3000 flights the LP in Vranas et al. (1994a) was solved faster. However, our solutions are for the most part already integral (the only instance where the solution was not integral was the 40-percent connectivity instance of the 1000-flight example). The total amount of time required to find an integral solution from the LP in Vranas et al. (1994a), found in the total time column, includes the time required to solve the LP relaxation, found in the LP Time column, plus the time required to perform a branch and bound heuristic. If we compare the

Table IV

Previous Results at the Infeasibility Border for 3,000 Flights

\begin{tabular}{ccccccc}
\hline$|\mathscr{F}|$ & $|\mathscr{G}| /|\mathscr{F}|$ & $\begin{array}{c}\text { Dep. } \\
\text { Capacity }\end{array}$ & $\begin{array}{c}\text { Arr. } \\
\text { Capacity }\end{array}$ & $\begin{array}{c}\text { LP } \\
\text { Time }\end{array}$ & $\begin{array}{c}\text { Total } \\
\text { Time }\end{array}$ & $\begin{array}{c}\% \\
\text { Nonint. }\end{array}$ \\
\hline 3,000 & 0.20 & $\infty$ & 12 & 1,453 & 11,360 & not given \\
3,000 & 0.40 & $\infty$ & 18 & 1,808 & 13,291 & not given \\
3,000 & 0.60 & $\infty$ & 17 & 2,547 & 17,980 & not given \\
3,000 & 0.80 & $\infty$ & 18 & 3,072 & 25,021 & not given \\
\hline
\end{tabular}


Table V

Results for Varying Capacity Levels for 1,000 Flights

\begin{tabular}{ccccccc}
\hline$|\mathscr{F}|$ & $|\mathscr{G}| /|\mathscr{F}|$ & $\begin{array}{c}\text { Dep. } \\
\text { Capacity }\end{array}$ & $\begin{array}{c}\text { Arr. } \\
\text { Capacity }\end{array}$ & $\begin{array}{c}\text { Obj. } \\
\text { Value }\end{array}$ & Time & $\begin{array}{c}\% \\
\text { Nonint. }\end{array}$ \\
\hline 1,000 & 0.20 & 32 & 17 & 50,750 & 342 & 0 \\
1,000 & 0.20 & 32 & 16 & 55,450 & 227 & 0 \\
1,000 & 0.20 & 32 & 15 & 63,525 & 262 & 0 \\
1,000 & 0.20 & 32 & 14 & inf & - & - \\
1,000 & 0.40 & 18 & 12 & 47,000 & 290 & 0 \\
1,000 & 0.40 & 18 & 10 & 79,916 & 521 & 2.2 \\
1,000 & 0.40 & 17 & 10 & 88,241 & 741 & 4 \\
1,000 & 0.40 & 16 & 10 & inf & - & - \\
1,000 & 0.60 & 20 & 18 & 22,316 & 369 & 0 \\
1,000 & 0.60 & 20 & 15 & 33,292 & 376 & 0 \\
1,000 & 0.60 & 20 & 14 & 39,266 & 359 & 0 \\
1,000 & 0.60 & 20 & 13 & inf & - & - \\
1,000 & 0.80 & 30 & 30 & 17,000 & 183 & 0 \\
1,000 & 0.80 & 20 & 20 & 28,250 & 283 & 0 \\
1,000 & 0.80 & 19 & 19 & inf & - & - \\
\hline
\end{tabular}

amount of time required to find an integral solution, we see a significant improvement in computational time.

Tables V and VI were constructed to demonstrate how computational time and integrality are affected by changes in the capacities, i.e., how well does the model perform when the capacities are not at the infeasibility border? These results suggest that the computational time did not change significantly at different capacity levels. For the one case in which the solution was not completely integral, (1,000 flights at 40-percent connectivity), increasing the capacity resulted in integral solutions.

Air Traffic Flow Management Problem Test Cases. We next performed experiments on a connected network of four airports: Boston Logan (BOS), NY LaGuardia (LGA), Washington National (DCA), and a node representing all other airports (X). (See Figure 6.) Three hypothetical sectors, surrounding LaGuardia, were also introduced into the model. Different flights would traverse these sectors while en route to LaGuardia depending on the origin of the flight. The three airports (BOS, LGA, DCA) and the three sectors were the only capacitated elements in the system. The other sectors were allocated

\section{Table VI}

Results for Varying Capacity Levels for 3,000 Flights

\begin{tabular}{ccccccc}
\hline$|\mathscr{F}|$ & $|\mathscr{C}| /|\mathscr{F}|$ & $\begin{array}{c}\text { Dep. } \\
\text { Capacity }\end{array}$ & $\begin{array}{c}\text { Arr. } \\
\text { Capacity }\end{array}$ & $\begin{array}{c}\text { Obj. } \\
\text { Value }\end{array}$ & $\begin{array}{c}\% \\
\text { Time }\end{array}$ & $\begin{array}{c}\% \\
\text { Nonint. }\end{array}$ \\
\hline 3,000 & 0.20 & 30 & 30 & 42,000 & 4,537 & 0 \\
3,000 & 0.20 & 20 & 20 & 228,000 & 5,475 & 0 \\
3,000 & 0.20 & 19 & 19 & inf & - & - \\
3,000 & 0.40 & 30 & 30 & 42,000 & 5,062 & 0 \\
3,000 & 0.40 & 20 & 20 & 234,000 & 4,703 & 0 \\
3,000 & 0.40 & 19 & 19 & inf & - & - \\
3,000 & 0.60 & 30 & 30 & 42,000 & 5,629 & 0 \\
3,000 & 0.60 & 20 & 20 & 234,000 & 5,407 & 0 \\
3,000 & 0.60 & 19 & 19 & inf & - & - \\
3,000 & 0.80 & 30 & 30 & 42,000 & 6,021 & 0 \\
3,000 & 0.80 & 20 & 20 & 252,000 & 9,411 & 0 \\
3,000 & 0.80 & 19 & 19 & inf & - & - \\
\hline
\end{tabular}

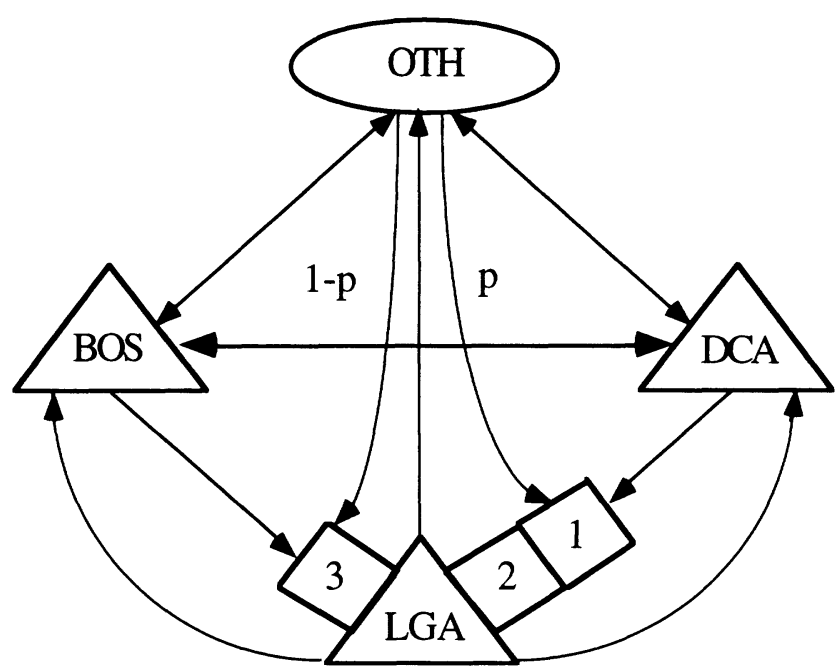

Figure 6. Sector flow model.

unlimited capacity. We performed one set of experiments for 200 flights over a 24-hour time period and another set for 1,000 flights over a 24-hour time period. The 200-flight dataset was obtained from the January 1993 Official Airline Guide (OAG). For the larger set of 1,000 flights, the data were generated by the Pseudo-OAG Generator (POAGG), which is flight generation software developed at Draper Laboratories that realistically mimics the flight schedules of the OAG. All models were programmed in GAMS, run on a Sun SPARCstation 10 model 41 and solved with the solver CPLEXMIP 2.1. For most of the test cases the time interval was five minutes long. Since some of the sectors could be crossed in under 15 minutes, we tried to select a time interval that would capture as many sectors as possible without becoming prohibitively large. With this in mind, we decided to use a five-minute interval whenever possible.

For the set of 200 flights, the time frame was 24 hours divided into discrete time units of five minutes each. To solve the problem CPLEX requires 234 seconds CPU time. Moreover, the resulting optimal solution was integral.

We were able to solve the 1000 flights problem at the infeasibility border over a 24 hour time period considering 15 minute intervals in 436 seconds CPU time. The optimal solution was once again integral. For the complete set of results see Table VII. Notice that the computation time in CPU seconds varies very little with the capacity restrictions in flights per time interval and that the solutions were completely integral.

Lastly, we obtained two realistic size datasets obtained directly from the OAG flight guide. This dataset has also been used to solve similar problems at the MITRE Corporation. The first dataset consists of 278 flights, 10 airports and 178 sectors, tested over a 7-hour time-frame with 5 -minute intervals. The second of these datasets consists of 1,002 flights, 18 airports, and 305 sectors tested over an 8 -hour time frame with 5 -minute intervals. 
Table VII

Results for Varying Capacity Levels for 1,000 Flights

\begin{tabular}{cccccc}
\hline $\begin{array}{c}\text { Sector } \\
\text { Capacity }\end{array}$ & $\begin{array}{c}\text { Dep. } \\
\text { Capacity }\end{array}$ & $\begin{array}{c}\text { Arr. } \\
\text { Capacity }\end{array}$ & $\begin{array}{c}\text { Obj. } \\
\text { Value }\end{array}$ & Time & $\begin{array}{c}\% \\
\text { Nonint. }\end{array}$ \\
\hline 50 & 20 & 20 & 31,975 & 425 & 0 \\
20 & 20 & 20 & 31,975 & 427 & 0 \\
20 & 10 & 10 & inf & - & - \\
15 & 15 & 15 & 68,725 & 427 & 0 \\
12 & 12 & 12 & 244,225 & 450 & 0 \\
11 & 11 & 11 & inf & - & - \\
10 & 12 & 12 & 24,225 & 456 & 0 \\
5 & 12 & 12 & 24,350 & 432 & 0 \\
4 & 12 & 12 & 250,975 & 466 & 0 \\
3 & 12 & 12 & 295,225 & 459 & 0 \\
2 & 12 & 12 & inf & - & - \\
\hline
\end{tabular}

The sector crossing times, sector and airport capacities, and required turnaround times were all provided by the FAA. Nothing used in these datasets was generated or hypothesized. We believe that these datasets are very comparable to the problem being solved everyday by the FAA.

For the first problem, consisting of 43,226 constraints and 18,733 variables, we found an optimal solution in 1,141 seconds. Furthermore, the solution obtained was completely integral. The second and larger dataset consisting of 151,662 constraints and 69,497 variables, was solved to optimality in 29,920 seconds, again achieving completely integral solutions.

In summary, to address the questions we raised in the beginning of Section 5 we remark:

1. In all but one instance in MAGHP and all instances of TFMP the relaxations of (MAGHP) and (TFMP) were integral.

2. The integrality of solutions was not affected by problem parameters, nor the size of the problem, except for the one instance in which the solution was nonintegral.

3 . The computational time required to obtain an optimal solution increases with the degree of connectivity as well as with the size of the problem.

4. Our approach improves upon earlier work particularly in obtaining integral solutions.

5. We are able to solve large, realistic size problems in a reasonable amount of time. In addition, because we were able to solve the two instances of the TFMP with real data, we are very optimistic that our approach can effectively address the TFMP. Indeed, the reason we did not solve bigger problems is the difficulty of obtaining real data and memory restrictions of the SPARCstation.

\section{CONCLUSIONS AND DIRECTIONS FOR FUTURE WORK}

We have presented what we believe is a realistic and practical approach to solve the Air Traffic Flow Management Problem. The TFMP model takes into account all the capacitated elements in the system (arrival, departure, and sector capacity) and easily extends to incorporate the dependence of airport runway capacity of departures and arrivals, hub connectivity, banking, and rerouting flights when capacity levels drop drastically.

The FAA has been operating for several years in Washington, D.C. an Air Traffic Control System Command Center (ATCSCC), equipped with outstanding informationgathering capabilities that dynamically keeps track of all the information about capacities, flight information, weather, etc. As we have mentioned earlier, the FAA uses a computerized procedure to allocate ground holding delays based on first-come-first-serve rule. We believe that the present optimization-based approach is well suited to be the optimization "brain" for this system. However, there are important issues that need to be addressed before applying an optimization based approach in a real world environment:

(a) Interaction with airlines. After the ground delays are issued, the airlines have the opportunity to propose modifications to these delays through a cancellation and substitution process. It would be interesting to analyze the effects of this interaction.

(b) Dynamic updating of decisions. Ground and enroute delays are both generated simultaneously several hours before a flight leaves. In practice, however, enroute delays are not issued until after the aircraft is in the air. Clearly more research is needed on the implications of issuing enroute delays on a much shorter time scale and on how to update the previous solution to incorporate any new available information.

(c) Stochastic modeling. The model presented in this paper assumes a deterministic environment. Clearly more research is needed to account for stochasticities inherent in a system that is dependent upon weather conditions.

Although we have presented our formulations in the context of air traffic control, we envision other applications of our models in any area in which goods are dynamically flowing through a system with several types of capacitated elements such as manufacturing and ground transportation systems.

\section{APPENDIX A. ON THE POLYHEDRAL RELATIONSHIPS BETWEEN GROUND HOLDING FORMULATIONS}

We intend to establish Proposition 1. Since $I P_{M A G H P}^{\prime}$, $I P_{V B O}$ and $I P_{T P}$ are valid integer programming formulations, it is clear that $I P_{M A G H P}^{\prime}=I P_{V B O}=I P_{T P}$. Moreover, since the IP is more restrictive than its relaxation, $I P_{M A G H P}^{\prime}$ $\subseteq P_{M A G H P}^{\prime}$.

To show the relationship $P_{M A G H P}^{\prime} \subseteq P_{T P}$ we will start with a feasible point in $P_{M A G H P}^{\prime}, \bar{z}_{f t}$, and show that this is indeed feasible to $P_{T P}$. The first two constraints and the last constraint are identical in the two models. So what remains to be shown is that any point, $\bar{z}_{f t}$, that satisfies the third constraint of $P_{M A G H P}^{\prime}$ will also satisfy the third constraint of $P_{T P}$. So 


$$
\begin{aligned}
& \sum_{t \in \mathscr{T}^{q}, t \leqslant \tau}\left(\bar{z}_{f t}-\bar{z}_{f, t-1}\right)-\sum_{t^{\prime} \in \mathcal{T}_{f}^{q}, t^{\prime} \leqslant \tau-s_{f}-\left(r_{f}-d_{f}\right)}\left(\bar{z}_{f^{\prime} t^{\prime}}-\bar{z}_{f^{\prime}, t^{\prime}-1}\right) \\
& =\bar{z}_{f \tau}-\bar{z}_{f^{\prime}, \tau-s_{f}-\left(r_{f}-d_{f}\right)}=\bar{z}_{f, t+\left(r_{f}-d_{f}\right)}-\bar{z}_{f, t-s_{f}} \leqslant 0 .
\end{aligned}
$$

So the point, $\bar{z}_{f t}$, satisfies the third constraint of $P_{T P}$ and all of the constraints of $P_{T P}$ hold. Thus, the point $\bar{z}_{f t}$ does indeed lie in the polyhedron $P_{T P}$. This establishes the relationship $P_{M A G H P}^{\prime} \subseteq P_{T P}$.

Now we need to prove the relationship $P_{T P} \subseteq P_{V B O}$. To show this we will start with a feasible point in $P_{T P}, \bar{z}_{f t}$, and show that this is indeed feasible to $P_{V B O}$. Once again, the first two constraints and the last constraint are identical in the two models. So what remains to be shown is that any point, $\bar{z}_{f t}$, that satisfies the third constraint of $P_{T P}$ will also satisfy the third and fourth constraints of $P_{V B O}$. So

$$
\begin{aligned}
g_{f}= & \sum_{t \in T_{f}^{a}} t\left(\bar{z}_{f t}-\bar{z}_{f, t-1}\right)-r_{f}, \\
g_{f^{\prime}}- & d_{f}+s_{f^{\prime}}+r_{f^{\prime}}-g_{f} \\
= & \sum_{t \in T_{f}^{a}} t\left(\bar{z}_{f^{\prime} t}-\bar{z}_{f^{\prime}, t-1}\right)-r_{f^{\prime}}-d_{f}+s_{f^{\prime}}+r_{f^{\prime}} \\
& -\sum_{t \in T_{f}^{a}} t\left(\bar{z}_{f t}-\bar{z}_{f, t-1}\right)-r_{f} \\
= & -\bar{z}_{f^{\prime}, r_{f}^{\prime}}-\ldots-\bar{z}_{f^{\prime}, r_{f}^{\prime}+\bar{T}_{f^{\prime}}-1}+r_{f^{\prime}}+\bar{T}_{f^{\prime}}-r_{f^{\prime}}-d_{f} \\
& +s_{f^{\prime}}+r_{f^{\prime}}+\bar{z}_{f, r_{f}}+\ldots+\bar{z}_{f, r_{f}+\bar{T}_{f}-1}-r_{f}-\bar{T}_{f}+r_{f} \\
= & -\bar{z}_{f^{\prime}, r_{f}^{\prime}}-\ldots-\bar{z}_{f^{\prime}, r_{f}^{\prime}+\bar{T}_{f^{\prime}}-1}+\bar{T}_{f^{\prime}}-d_{f}+s_{f^{\prime}}+r_{f^{\prime}} \\
& +\bar{z}_{f, r_{f}}+\ldots+\bar{z}_{f, r_{f}+\bar{T}_{f}-1}-\bar{T}_{f} \\
\leqslant & -\left(r_{f^{\prime}}+\bar{T}_{f^{\prime}}-1-\left(r_{f}-s_{f^{\prime}}-\left(r_{f}-d_{f}\right)\right)+1\right)+\bar{T}_{f^{\prime}} \\
& -d_{f}+s_{f^{\prime}}+r_{f^{\prime}}+\left(\bar{T}_{f}+r_{f}-1-r_{f}+1\right)-\bar{T}_{f} \\
= & -\left(r_{f^{\prime}}+\bar{T}_{f^{\prime}}-r_{f}+s_{f^{\prime}}+r_{f}-d_{f}\right)+\bar{T}_{f^{\prime}}-d_{f}+s_{f^{\prime}} \\
& +r_{f^{\prime}}+0 \\
= & -r_{f^{\prime}}-s_{f^{\prime}}+d_{f}-d_{f}+s_{f^{\prime}}+r_{f^{\prime}}=0,
\end{aligned}
$$

where $\bar{T}_{f}$ is maximum amount of time that flight $f$ may arrive late, so all the constraints hold and the point $\bar{z}_{f t}$ does indeed lie in the polyhedron $P_{V B O}$. This establishes the relationship $P_{T P} \subseteq P_{V B O}$.

\section{APPENDIX B. ON THE NONINTEGRALITY OF THE POLYHEDRON $\boldsymbol{P}_{\text {MAGHP }}$}

In this section we prove Theorem 1a, i.e., the polyhedron $P_{M A G H P}$ is not integral, by providing the following example which has a fractional extreme point. Consider the case in which there are two flights arriving and being continued by two flights departing from a given airport during a restricted time window. The data of the problem is as follows:

$|\mathscr{K}|=1, \quad \mathscr{T}=\{1,2,3,4\}, \quad \mathscr{C}=\{(1,1),(2,2)\}$,

i.e., the arriving flight $i$ is continued by departing flight $i$. The turnaround times are

$s_{1}=0, s_{2}=1$.
The time windows are:

$T_{1}^{a}=\{1,2\}, \quad T_{2}^{a}=\{1,2\}, \quad T_{1}^{d}=\{1,2\}, \quad T_{2}^{d}=\{2,3\}$.

Notice that flight 2 can depart only during time slots 2 and 3 , since the turnaround time for the second flight is 1 . The decision variables are:

$y_{11}, y_{12}, y_{22}, y_{23}, z_{11}, z_{12}, z_{21}, z_{22}$,

with the interpretation that $y_{i j}=1$ if flight $i$ departs by time $j$ and $z_{i j}=1$ if flight $i$ arrives by time $j$. Because of the time windows,

$y_{13}=1, \quad y_{24}=1, \quad z_{13}=1, \quad z_{23}=1$.

The capacities are:

$D(1)=D(2)=D(3)=1, \quad A(1)=A(2)=A(3)=1$.

The resulting formulation $(M A G H P)$ is:

$y_{11} \leqslant 1, \quad y_{12}-y_{11}+y_{22} \leqslant 1, \quad 1-y_{12}+y_{23}-y_{22} \leqslant 1$,

$z_{11}+z_{21} \leqslant 1, \quad z_{12}-z_{11}+z_{22}-z_{21} \leqslant 1$,

$1-z_{12}+1-z_{22} \leqslant 1, \quad y_{12}-y_{11} \geqslant 0$,

$y_{23}-y_{22} \geqslant 0, \quad z_{12}-z_{11} \geqslant 0$,

$z_{22}-z_{21} \geqslant 0, \quad y_{11}-z_{11} \leqslant 0, \quad y_{12}-z_{12} \leqslant 0$,

$y_{22}-z_{21} \leqslant 0, \quad y_{23}-z_{22} \leqslant 0$.

Letting

$x=\left(y_{11}, y_{12}, y_{22}, y_{23}, z_{11}, z_{12}, z_{21}, z_{22}\right)^{\prime}$ and $b$

$=(1,1,0,1,1,-1,0,0,0,0,0,0,0,0)^{\prime}$,

and

$$
A=\left|\begin{array}{cccccccc}
y_{11} & y_{12} & y_{22} & y_{23} & z_{11} & z_{12} & z_{21} & z_{22} \\
1 & 0 & 0 & 0 & 0 & 0 & 0 & 0 \\
-1 & 1 & 1 & 0 & 0 & 0 & 0 & 0 \\
0 & -1 & -1 & 1 & 0 & 0 & 0 & 0 \\
0 & 0 & 0 & 0 & 1 & 0 & 1 & 0 \\
0 & 0 & 0 & 0 & -1 & 1 & -1 & 1 \\
0 & 0 & 0 & 0 & 0 & -1 & 0 & -1 \\
1 & -1 & 0 & 0 & 0 & 0 & 0 & 0 \\
0 & 0 & 1 & -1 & 0 & 0 & 0 & 0 \\
0 & 0 & 0 & 0 & 1 & -1 & 0 & 0 \\
0 & 0 & 0 & 0 & 0 & 0 & 1 & -1 \\
1 & 0 & 0 & 0 & -1 & 0 & 0 & 0 \\
0 & 1 & 0 & 0 & 0 & -1 & 0 & 0 \\
0 & 0 & 1 & 0 & 0 & 0 & -1 & 0 \\
0 & 0 & 0 & 1 & 0 & 0 & 0 & -1
\end{array}\right|,
$$

the feasible space can be written as $A x \leqslant b$.

Notice that matrix $A$ is not totally unimodular since the submatrix consisting of the columns corresponding to the variables $y_{12}, y_{22}, z_{12}$, and $z_{21}$ and the third, fifth, twelfth, and thirteenth rows: 


$$
\begin{aligned}
& y_{12} \quad y_{22} \quad z_{12} \quad z_{21} \\
& \left(\begin{array}{cccc}
-1 & -1 & 0 & 0 \\
0 & 0 & 1 & -1 \\
1 & 0 & -1 & 0 \\
0 & 1 & 0 & -1
\end{array}\right),
\end{aligned}
$$

has determinant of 2 . The objective function

Min $2 y_{11}-4 y_{12}+2 y_{22}-6 y_{23}-3 z_{11}+6 z_{12}-3 z_{21}$

$$
+6 z_{22}
$$

gives an optimal solution of

$y_{11}=0 \quad y_{22}=0 \quad z_{11}=\frac{1}{2} \quad z_{21}=\frac{1}{2}$

$y_{12}=\frac{1}{2} \quad y_{23}=\frac{1}{2} \quad z_{12}=\frac{1}{2} \quad z_{22}=\frac{1}{2}$,

which shows that the polyhedron $P_{M A G H P}$ is not integral. Furthermore, this is the objective function that is obtained when we let $c_{f}^{g}=1, c_{f}^{a}=3$ for all $f \in \mathscr{F}$. So, even with the restriction that $c_{g}=c_{f}^{g}$ and $c_{a}=c_{f}^{a}$ for all $f \in \mathscr{F}$, the polyhedron $P_{M A G H P}$ is not integral.

\section{APPENDIX C. FACET DEFINING CONSTRAINT PROOFS}

In this section we analyze the polyhedral structure of the $\operatorname{conv}\left(I P_{M A G H P}\right)$ and provide the proof of the first half of Theorem $1 \mathrm{~b}$ that establishes which constraints are facets of $\operatorname{conv}\left(I P_{M A G H P}\right)$. The proof of the second half of Theorem $1 \mathrm{~b}$ concerning problem (TFMP) is similar, but more algebraically involved. We first show that the constraint

$$
\sum_{f: t \in T_{f}^{d}}\left(y_{f t}-y_{f, t-1}\right) \leqslant D_{k}(t) \quad \forall k \in \mathscr{H}, t \in \mathscr{F},
$$

is not a facet of $\operatorname{conv}\left(I P_{M A G H P}\right)$ by constructing a counterexample with two flights, one arriving at airport $k$ and one departing from airport $k$, three time periods and $D(t)=1$, $A(t)=1$. Then only the variables $y_{11}, y_{12}, y_{13}, z_{11}, z_{12}$, and $z_{13}$ are defined. The complete set of feasible solutions to $I P_{M A G H P}$ is given by:

$$
\begin{aligned}
& \begin{array}{llllll}
y_{11} & y_{12} & y_{13} & z_{11} & z_{12} & z_{13}
\end{array} \\
& \left(\begin{array}{llllll}
0 & 0 & 0 & 0 & 0 & 0 \\
0 & 0 & 0 & 0 & 0 & 1 \\
0 & 0 & 0 & 0 & 1 & 1 \\
0 & 0 & 0 & 1 & 1 & 1 \\
0 & 0 & 1 & 0 & 0 & 1 \\
0 & 0 & 1 & 0 & 1 & 1 \\
0 & 0 & 1 & 1 & 1 & 1 \\
0 & 1 & 1 & 0 & 1 & 1 \\
0 & 1 & 1 & 1 & 1 & 1 \\
1 & 1 & 1 & 1 & 1 & 1
\end{array}\right) .
\end{aligned}
$$

In this case, $\operatorname{dim}\left(I P_{M A G H P}\right)=5$ which can be determined by checking the rank of the matrix of solutions. We define the set

$$
H_{t}=\left\{(y, z) \in I P_{M A G H P}: \sum_{f: t \in T^{\phi}}\left(y_{f t}-y_{f, t-1}\right)=1\right\}
$$

for some $t \in \mathscr{T}$.
Then, $H_{3}=\{(0,0,1,0,0,1),(0,0,1,0,1,1),(0,0,1,1$, $1,1)\}$. In this case, the maximum number of affinely independent points in $H_{3}$ is less than the $\operatorname{dim}\left(I P_{M A G H P}\right)-1$. We conclude that the constraint $\sum_{f: t \in T_{f}^{d}}\left(y_{f t}-y_{f, t-1}\right) \leqslant$ $D_{k}(t), \forall k \in \mathscr{K}, t \in \mathscr{F}$ is not a facet. The same result can be checked in a similar manner for the constraint $\sum_{f: t \in T_{a}}$ $\left(z_{f t}-z_{f, t-1}\right) \leqslant A_{k}(t), \forall k, t$.

For ease of exposition we consider instances of $(M A G H P)$ such that

- $\left|T_{f}\right|$ is that same for all $f$ and therefore $D=\max _{f}\left|T_{f}\right|=$ $\left|T_{f}\right|$,

- $s_{f}=0, \forall f \in \mathscr{F}$,

- $A_{k}(t), D_{k}(t) \geqslant 1, \forall k, t$.

We consider an instance of $(M A G H P)$ with $|\mathscr{F}|$ flights in which $|\mathscr{G}|(<|\mathscr{F}|)$ of these flights are continued. These flights were arranged such that the first $|\mathscr{C}|$ flights are continued by flights $|\mathscr{C}|+1, \ldots, 2|\mathscr{C}| \leqslant|\mathscr{F}|$, with flight 1 being followed by flight $|\mathscr{C}|+1$, flight 2 being followed by flight $|\varphi|+2$, and so on.

We first determine $\operatorname{dim}\left(I P_{M A G H P}\right)$ by constructing the following matrix of solutions, in which each row represents a solution to $(M A G H P)$ (see Figure 7). The rows of this matrix are affinely independent and there are $2|\mathscr{F}| D+1$ such rows. So, we have exhibited $2|\mathscr{F}| D+1$ affinely independent points in $I P_{M A G H P}$ and thus, $\operatorname{dim}\left(I P_{M A G H P}\right)=$ $2|\mathscr{F}| D$.

We next consider the set

$G_{f t}=\left\{(y, z) \in I P_{M A G H P}: y_{f t}-y_{f, t-1}=0\right\}$

for some $f \in \mathscr{F}, t \in \mathscr{T}$.

If $f \in\{1, \ldots,|\mathscr{C}|\}$, then there are four distinct solutions from the matrix of Figure 7 that do not belong to $G_{f t}$. For each of these rows, replace the 0 in the $y_{f, t-1}$ column with an 1. If $f \in\{|\mathscr{G}|+1, \ldots, 2|\mathfrak{G}|\}$ then there are two distinct solutions from Figure 7 that do not belong to $G_{f t}$. For each of these rows, replace the 1 in the $y_{f, t}$ column with a 0 . If $f \in\{2|\mathscr{G}|+1, \ldots,|\mathscr{F}|\}$ then there are two unique solutions from Figure 7 which do not belong to $G_{f t}$. For each of these rows, replace the 0 in the $y_{f, t-1}$ column with a 1 . For all of these cases, we have constructed a matrix with $|\mathscr{F}| D$ affinely independent rows, proving that $\operatorname{dim}\left(G_{f t}\right)$ $\geqslant|\mathscr{F}| D-1$. Since $G_{f t}$ is a proper face of $I P_{M A G H P}$, we know that $\operatorname{dim}\left(G_{f t}\right)<\operatorname{dim}\left(I P_{M A G H P}\right)$. So, $\operatorname{dim}\left(G_{f t}\right)=$ $|\mathscr{F}| D-1$ and thus, $G_{f t}$ is a facet of $I P_{M A G H P}$.

We next consider the set

$K_{f t}=\left\{(y, z) \in I P_{M A G H P}: z_{f t}-z_{f, t-1}=0\right\}$

for some $f \in \mathscr{F}, t \in \mathscr{T}$.

If $f \in\{1, \ldots,|\mathscr{G}|\}$ then there are three distinct solutions from the matrix of Figure 7 that do not belong to $G_{f t}$. For each of these rows, replace the 1 in the $y_{f, t}$ column with a 0 . If $f \in\{|C|+1, \ldots,|F|\}$, then there is only one distinct solution from Figure 7 that does not belong to $G_{f t}$, so remove this row. For each of these cases, we have constructed a matrix with $|\mathscr{F}| D$ affinely independent rows, 

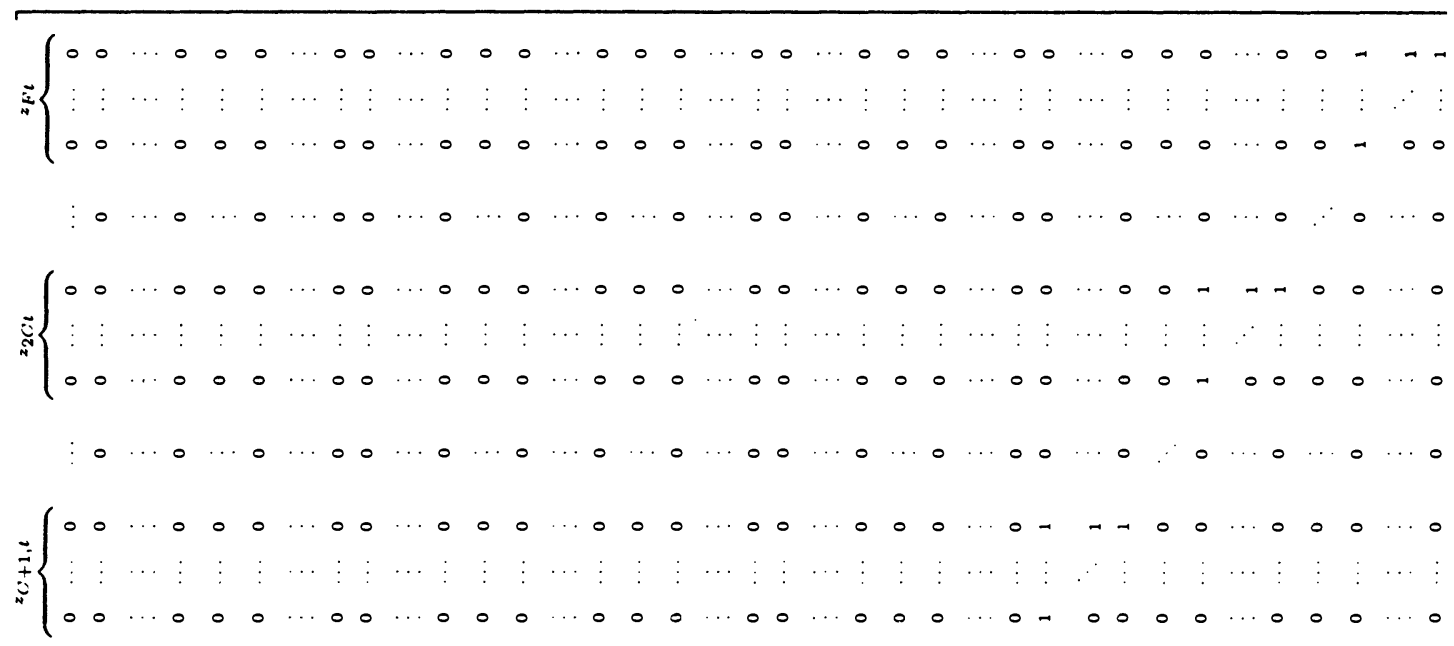

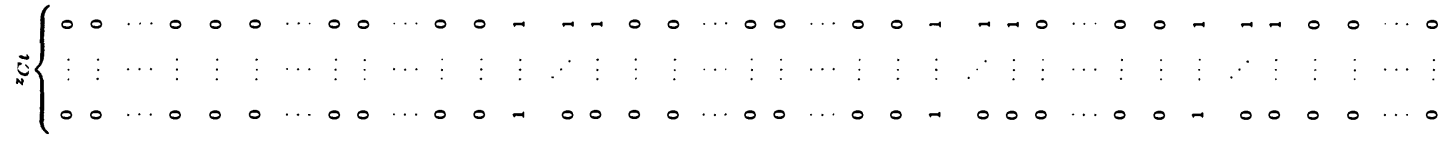

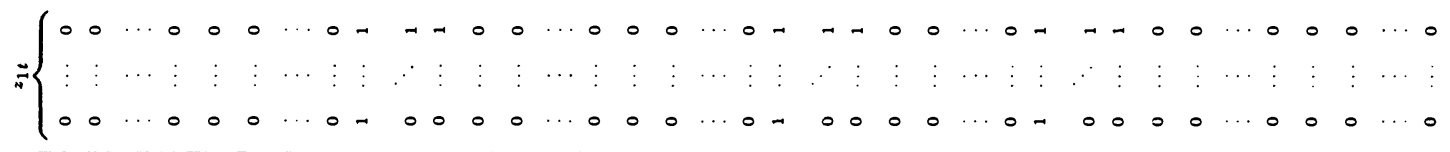

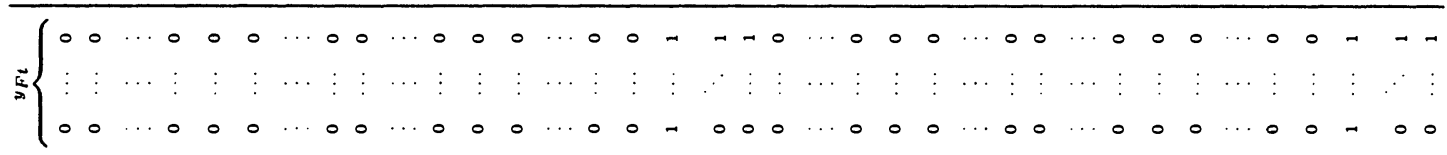

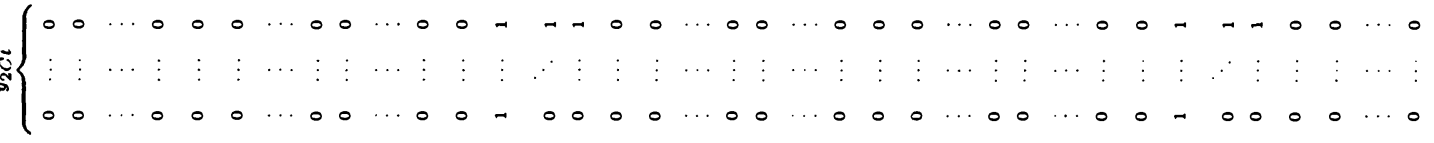

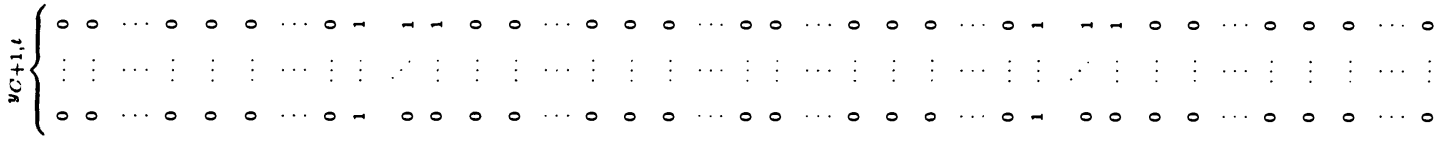

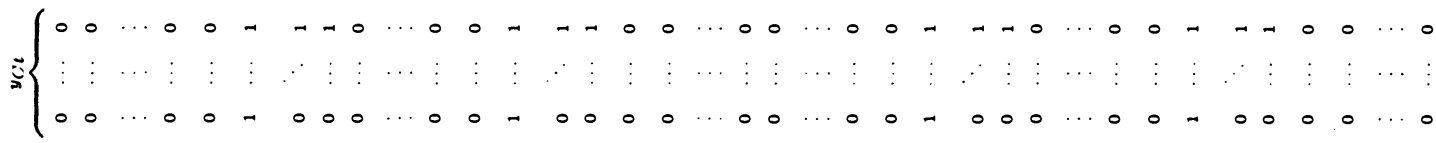

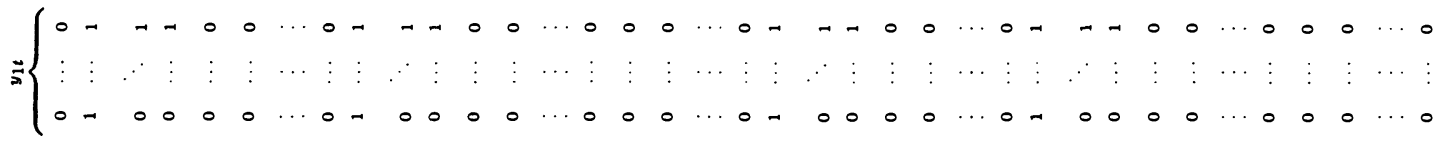

Figure 7. Matrix of Solutions to $I P_{M A G H P}$

proving that $\operatorname{dim}\left(K_{f t}\right) \geqslant|\mathscr{F}| D-1$. Since $K_{f t}$ is a proper face of $I P_{M A G H P}$, we know that $\operatorname{dim}\left(K_{f t}\right)<\operatorname{dim}\left(I P_{M A G H P}\right)$. So, $\operatorname{dim}\left(K_{f t}\right)=|\mathscr{F}| D-1$ and thus, $K_{f t}$ is a facet of $I P_{\text {MAGHP. }}$
We next consider the set

$M_{f t}=\left\{(y, z) \in I P_{M A G H P}: z_{f t}-y_{f, t-\left(r_{f}-d_{f}\right)}=0\right\}$ for some $f \in \mathscr{F}, t \in \mathscr{T}$. 
For all $f \in\{1, \ldots,|\mathscr{F}|\}$ there are $t-\underline{T}_{f}+1$ distinct solutions from the matrix of Figure 7 that do not belong to $M_{f t}$. For each of these rows replace the $0 \mathrm{~s}$ in the columns corresponding to $z_{f t^{\prime}}, t \leqslant t^{\prime} \leqslant \bar{T}_{f}$ with 1 s. $\bar{T}_{f}$ and $\underline{T}_{f}$ are the last possible and the earliest possible times that flight $f$ could arrive, respectively. The remaining matrix will have $|\mathscr{F}| D$ affinely independent rows, proving that $\operatorname{dim}\left(M_{f t}\right) \geqslant$ $|\mathscr{F}| D-1$. Since $M_{f t}$ is a proper face of $I P_{M A G H P}$, we know that $\operatorname{dim}\left(M_{f t}\right)<\operatorname{dim}\left(I P_{\text {MAGHP }}\right)$. So, $\operatorname{dim}\left(M_{f t}\right)=|\mathscr{F}| D-1$ and thus, $M_{f t}$ is a facet of $I P_{M A G H P}$.

Finally, we consider the set

$N_{f^{\prime} t}=\left\{(y, z) \in I P_{M A G H P}: y_{f t}-z_{f^{\prime} t}=0\right\}$

for some $\left(f^{\prime}, f\right) \in \mathscr{C}, t \in \mathcal{T}$.

For all $f \in\{1, \ldots,|\mathscr{F}|\}$ there are $t-\underline{T}_{f}+1$ distinct solutions from the matrix of Figure 7 that do not belong to $N_{f^{\prime} f t}$. For each of these rows replace the $0 \mathrm{~s}$ in the columns corresponding to $y_{f t^{\prime}}, t \leqslant t^{\prime} \leqslant \bar{T}_{f t}$ with $1 \mathrm{~s}$. The remaining matrix will have $|\mathscr{F}| D$ affinely independent rows, proving that $\operatorname{dim}\left(N_{f^{\prime} t t}\right) \geqslant|\mathscr{F}| D-1$. Since $N_{f^{\prime} f t}$ is a proper face of $I P_{M A G H P}$, we know that $\operatorname{dim}\left(N_{f^{\prime} f t}\right)<$ $\operatorname{dim}\left(I P_{M A G H P}\right)$. So, $\operatorname{dim}\left(N_{f^{\prime} f t}\right)=|\mathscr{F}| D-1$ and thus, $N_{f^{\prime} f t}$ is a facet of $I P_{M A G H P}$.

\section{ACKNOWLEDGMENTS}

We would like to thank Professor Amedeo Odoni for inspiring our work in this area and being a constant source of guidance and encouragement during the course of this research. We would like to thank Dr. David Winer and Dr. Thomas Mifflin of the FAA for encouraging and supporting our work. We would like to thank Dr. Kenneth Lindsay for providing data for some of the computational experiments, Ms. Wandy Sae-tan for performing some of the computational experiments and Dr. Eugene Gilbo for providing some of the figures. This research was supported in part by grants from Draper Laboratory and the FAA and by a Presidential Young Investigator Award DDM-9158118.

\section{REFERENCES}

Andreatta, G., A. R. Odoni, and O. Richetta. 1993. Models for the Ground-Holding Problem. In Large-Scale Computation and Information Processing in Air Traffic Control. L. Bianco and A. R. Odoni (ed.), Springer-Verlag, Berlin.

BALl, M. 1993. Personal Communication.

Garey, M. R. AND D. S. Johnson. 1979. Computers and Intractibility: A Guide to the Theory of NP-Completeness. W. H. Freeman \& Company, New York.

GILBO, E. P. 1993. Airport Capacity: Representation, Estimation, Optimization. IEEE Trans. Control Systems Technology, 1, 3, 144-154.

Helme, M. 1992. Reducing Air Traffic Delay in a Space-Time Network. Proc. 1992 IEEE International Conf. Systems, Man and Cybernetics, Chicago, 236-242.

Lindsay, K. S., E. A. Boyd, and R. Burlingame. 1993. Traffic Flow Management Modeling with the Time Assignment Model. Air Traffic Control Quarterly, 1, 3.

Nemhauser, G. AND L. Wolsey. 1988. Integer and Combinatorial Optimization. John Wiley \& Sons, New York.

Odoni, A. R. 1987. The Flow Management Problem in Air Traffic Control. In Flow Control of Congested Networks. A. R. Odoni and G. Szego (eds.), Springer-Verlag, Berlin.

Richetta, O. AND A. R. Odoni. 1993. Solving Optimally the Static Ground-Holding Policy Problem in Air Traffic Control. Transp. Sci. 27, 228-238.

Richetta, O. AND A. R. Odoni. 1994. Dynamic Solution to the Ground-Holding Policy Problem in Air Traffic Control. Transp. Res. 28a, 167-185.

$\rightarrow$ Terrab, M. And A. R. Odoni. 1993. Strategic Flow Control on an Air Traffic Network. Opns. Res. 41, 138-152.

Terrab, M. and S. Paulose. 1993. Dynamic Strategic and Tactical Air Traffic Flow Control. RPI Technical Report.

Vranas, P., D. Bertsimas, and A. R. Odoni. 1994a. The Multi-Airport Ground-Holding Problem in Air Traffic Control. Opns. Res. 42, 249-261.

Vranas, P., D. Bertsimas, and A. R. Odoni. 1994b. Dynamic Ground-Holding Policies for a Network of Airports. Transp. Sci. 28, 275-291.

WALD, M. L. 1995. Austere Future Looms for F.A.A. New York Times, October 15, A1. 